Original Research Paper

\title{
Investigation of the Fatty Acid Profile in Some Macroalgae in Relation to the Environmental Conditions for Biodiesel Production
}

\author{
${ }^{1}$ Nayrah Abdel Naby Shaltout and ${ }^{2}$ Nihal Galal Shams El-Din \\ ${ }^{I}$ Marine Chemistry Laboratory, Department of Marine Environment, \\ National Institute of Oceanography and Fisheries, Egypt \\ ${ }^{2}$ Hydrobiology Laboratory, Department of Marine Environment, \\ National Institute of Oceanography and Fisheries, Egypt
}

\author{
Article history \\ Received: 17-06-2015 \\ Revised: 07-09-2015 \\ Accepted: 11-11-2015 \\ Corresponding Author: \\ Nihal Galal Shams El-Din \\ Hydrobiology Laboratory, \\ Department of Marine \\ Environment, National Institute of \\ Fisheries and Aquatic Research, \\ Egypt \\ Email: nihalshamseldin@yahoo.com
}

\begin{abstract}
In this study, fatty acids profile was determined in four algal species; namely Ulva fasciata (Chlorophyta), Corallina mediterranea, Corallina officinalis and Pterocladiella capillacea (Rhodophyta) which were collected from two sites (Abu-Qir Bay and Eastern Harbor) located on the Egyptian Mediterranean Sea during the four seasons in the period (2011-2012). In addition, nutrient content in the algal species (total nitrogen and phosphorus) and the environmental conditions of the ambient sea water were measured to find out their effect on the algal fatty acids content. The nitrogen content in algal species was almost equal in both sites. In contrast, the phosphorus content was higher in Abu-Qir (0.33$1.71 \%)$ than in Eastern Harbor (0.21-0.74\%). The fatty acids profile showed different correlations with water's physico-chemical properties. The fatty acids profile of the four algal species revealed high content of SFAs against MUFA and PUFA in both sites. Consequently, the algae were screened to define the suitable site, season and species for the highest quality and quantity of biodiesel production. Biodiesel physical properties; the Degree of Unsaturation (DU), Long Chain Saturation Factor (LCSF), Cold Filter Plugging Point (CFPP), Iodine Value (IV), Saponification Value (SV), the Cetane Number (CN), Kinematic viscosity (v), density $(\rho)$, the Higher Heating Value (HHV), C18:3\% (wt\%) and double bond $\mathrm{Db}$ $\geq 4(\mathrm{wt} \%)$ were calculated. All the investigated algae showed biodiesel properties that follow the standard values set for optimum biodiesel, with the green alga $U$. faciata collected from Abu-Qir during winter, as the best choice for producing biodiesel; whereas, the extracted fatty acids from Corallina officinalis is more suitable to be used for production of blenders, which can be added to the petroleum diesel in order to increase its quality, oxidative stability and octane number.
\end{abstract}

Keywords: Fatty Acids Profile, Algae, Nutrient Content, Environmental Parameters, Biodiesel

\section{Introduction}

The growing concern on depletion of fossil fuels and their environmental effects of burning, particularly greenhouse gas emissions and consequently climate change, have led to search for viable renewable fuel alternatives, which should be eco-friendly fuel unlike petrodiesel (Trivedi et al., 2013). Biodiesel is an alternative liquid fuel produced by a chemical reaction between a vegetable oil or animal fat with a short chain alcohol, called transesterification producing fatty acid methyl ester, where it can be used in diesel engines blended with standard gasoil or alone (Marchetti et al., 2007). From 
an environmental point of view, biodiesel has several benefits such as the reduction of carbon monoxide $(50 \%)$ and carbon dioxide emissions (78\%). In addition it is a biodegradable, renewable and nontoxic and its use, instead of diesel, reduces fossil fuels consumption (Surendhiran and Vijay, 2014).

Actually, the most widely available forms of biofuel were obtained from corn starch, sugar cane, palm, oilseed rape and soybean. However, these traditional feedstocks for biodiesel are also feed for livestock on farms, as well as a valuable source of food for humans. Because of this, using them in biofuel production increases food crises and increases expenses for both the food and the fuel produced (Abishek et al., 2014). Also, these terrestrial crops may contribute to net greenhouse gas emission and may cause other environmental impacts such as freshwater reducing resources and changing in the water cycle (Demirbas, 2009). Whereas, using algae as a source of biodiesel can alleviate these problems in a number of ways. Firstly, many algae are not used as a primary food source for humans, meaning that it can be used solely for fuel production and there would be little impact in the food industry (Demirbas, 2009). Secondly, many of the waste-product extracts produced during the processing of algae for biofuel can be used as a sufficient animal feed. This is an effective way to minimize waste and a much cheaper alternative to the more traditional corn or grain based feeds (Pienkos and Darzins, 2009). Moreover, they are more productive than terrestrial plants and can succeed in salty or brackish water with only sunlight and available nutrients; they do not need any chemical fertilizers. For all these reasons, recent researches around the world focused on algae for biofuel production; either bioehanol (Goh and Lee, 2010), biogas (Hughes et al., 2012), or biodiesel (Maceiras et al., 2011).

In fact, marine algae are reported to be rich in soluble dietary fibers, carbohydrates, proteins, minerals, vitamins, antioxidants, phytochemicals and polyunsaturated fatty acids with a low caloric value (Polat and Ozugul, 2013). Singh and Gu (2010); John and Anisha (2011) reported that, macroalgal biomass can store large amounts of oil which can be exploited for the production of biodiesel. They are remarkably affected by external factors such as geographical location, environment, season and sampling conditions (Renaud and Luong-Van, 2006).

Fatty acids are one of the most important components in seaweeds. They are classified in three groups; Saturated Fatty Acids (SFA), Monounsaturated Fatty Acids (MUFAs) and Polyunsaturated Fatty Acids (PUFAs) (Polat and Ozogul, 2013). The PUFAs and MUFAs are of particular interest, because they are very important for human health maintenance and they are synthesized only by plants (Yang et al., 2000; Pulz and Gross, 2004; Le Goff et al., 2013). In contrast, high concentrations of SFA in dietary sources are undesirable, since they have negative effects on human health (Muralidhar et al., 2010), but this group is of special interest in biofuel production (Islam et al., 2013). Many previous researches were concerned with the production of biofuel from a large number of macroalgae such as Ascophyllum nodosum, Codium tomentosum, Enteromorpha intestinalis, Ulva rigida and others (Maceiras et al., 2011). In fact, the first step in developing an algal process is to choose the algal species. Pulz and Gross (2004) observed that successful algal biotechnology mainly depends on choosing the right alga with relevant properties for specific culture conditions and products.

However, the properties of biodiesel depend on the type of fatty acids and their saturation levels. Suitability of the biodiesel as a diesel engine fuel is determined by its physical properties to know its feasibility as a diesel engine fuel (Saravanan and Chandrasekar, 2013).

In Egypt, seaweeds grow luxuriantly along the Mediterranean coast and many species are still unexploited, despite their great importance ecologically, economically and industrially (Shams El-Din and El-Sherif, 2012). Most of the literature were interested in seaweeds in medicinal uses (Matloub et al., 2013; El-Kassas and Attia, 2014), antifouling agents (Tadros et al., 2006), bioremediation agents (Abdallah, 2013) and nutritional sources for fish (Wassef et al., 2013) and for human (Shams El-Din and El-Sherif, 2012). However, very scarce studies were carried on seaweeds as a source for biofuel production and the only available literature of Afify et al. (2010) has investigated neither the effect of environmental conditions, nor the effect of seasonal variations on biochemical and fatty acids production.

This study aimed to evaluate the potential of different seaweeds for biodiesel production and the impact of the spatial and temporal variations on biodiesel quality. In order to achieve our aim, the study is subdivided into two parts; the first is the determination of the total nutrients contents (nitrogen and phosphorus) in seaweeds in addition to the environmental conditions of the ambiant sea water. The second is to determine the quality and quantity of produced biodiesel from different seaweeds at different seasons.

\section{Materials and Methods}

\section{The Study Area}

Water and algal samples were collected during 20112012 from two sites located on the Mediterranean Sea, Abu-Qir Bay and Eastern Harbor, which were chosen representing different environmental habitats along the Alexandria coast (Fig. 1). 


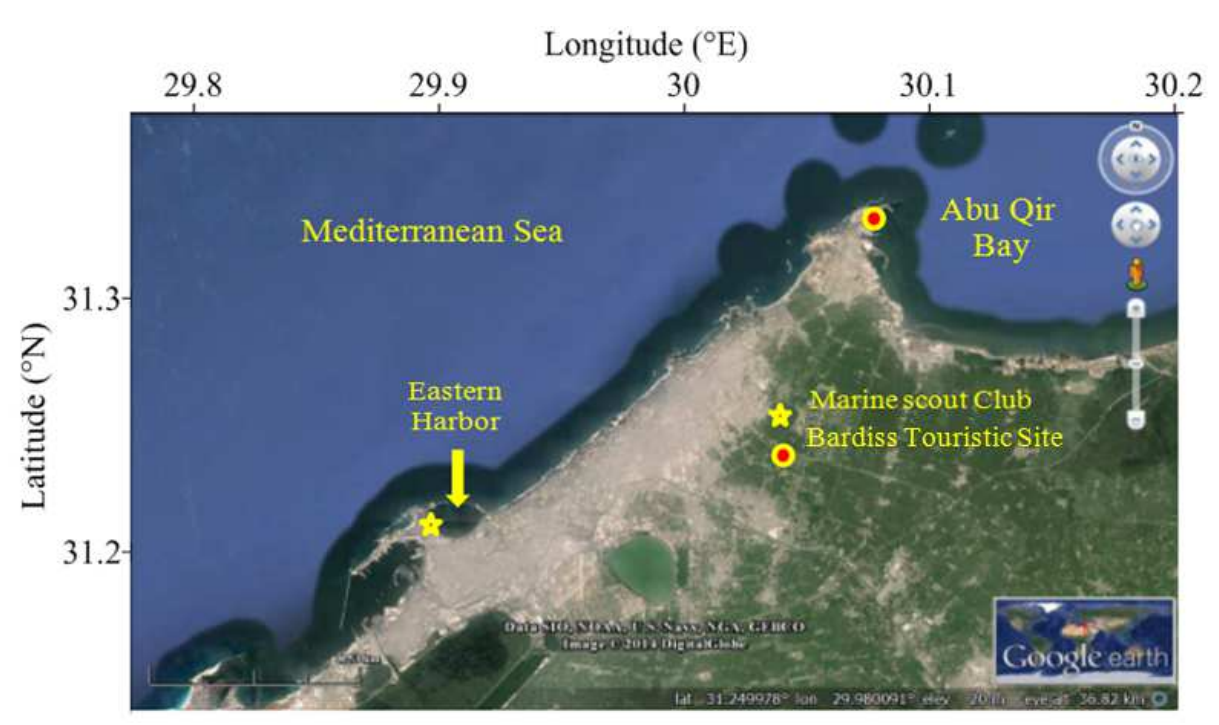

Fig. 1. The study area and sampling station during 2011-2012

The samples were collected from the beach of the touristic site "Bardiss" located at the extremely western head of Abu Qir Bay (A.Q.) at longitudes $30^{\circ} 07^{\prime} \mathrm{E}$ and latitudes $31^{\circ} 16^{\prime} \mathrm{N}$. The bay is a hot spot area since it is subjected properly to discharge of primary treated sewage water, in addition to fishing activities. As well as, a discharge of industrial wastewater through "Tabia Pumping Station" and the agricultural effluents from Lake Edku (Shams El-Din et al., 2014). This area is affected by the eastward windinduced current (El-Tawil and Khalil, 1983). However, A.Q. site is subjected to wave action and is characterized by exposed rocks extending $100 \mathrm{~m}$ from the coast. At the western edge, the substrate consists of chains of natural rocks surrounded by pools (Ismael, 2012). These rocks provide excellent substrata for a rich algal flora (EL-Sarraf, 1995).

The Eastern Harbor of Alexandria (E.H.) is a shallow, semi-enclosed embayment covering an area of about $2.8 \mathrm{~km}^{2}$, located along the central part of Alexandria. The southern part of the harbor has been reinforced by concrete blocks and the northern side is protected by an artificial breakwater with two inlets, an eastern and a western inlet. It is bordered to the east by a land projection, El-Silsila and to the northwest by a long causeway (El-Sayed and Khadr, 1999). The bay has always been the recipient of large volumes of domestic waste water from several point sources. In 1996-1997, however, all outfalls except one were closed. The same volume of waste water is now being disposed of through the remaining outfall on the south-west bay margin (Ismael, 2012). In addition, the bay is exposed to pollution due to intensive shipping and fishing activities. The algal samples were collected from the Scout club located in E.H. at $31^{\circ} 13.3^{\prime} \mathrm{N}$ latitude and $29^{\circ} 53.10^{\circ} \mathrm{E}$ longitude. However, the site sheltered four or five common seaweeds, which were recorded previously (Shams ElDin et al., 2007) and were found along the Egyptian Mediterranean coast.

\section{Sample Collection}

\section{Water Samples}

Water samples were collected seasonally, from the same sites and depths of the algae. The samples were collected directly into 5 liters capacity plastic Jerrycan, which were used for determinations of the studied parameters: Salinity, temperature, nitrite $\left(\mathrm{NO}_{2}\right)$, nitrate $\left(\mathrm{NO}_{3}\right)$, reactive soluble phosphate $\left(\mathrm{PO}_{4}\right)$, silicate $\left(\mathrm{SiO}_{4}\right)$ and oxidizable organic matter (O.M). pH was measured in situ using a Metrohm $(827 \mathrm{pH}) \mathrm{pH}$ electrode calibrated with TRIS buffer on total scale $\left(\mathrm{pH}_{\mathrm{T}}\right)$ following Dickson et al. (2007). Measurements of salinity was done using a Bekman Induction Salinometer (Model RS-7C), which is standardized with Standard Seawater Copenhagen, Denmark, of chlorinity $39.377 \mathrm{~g} \mathrm{l}^{-1}$. The conductivity ratio was measured to the nearest $1 \times 10^{-5}$ and converted to salinity, up to the nearest $0.01 \mathrm{~g} \mathrm{l}^{-1}$ using the tables of salinity/conductivity conversions. The temperature of A.Q. during the four seasons was measured using an ordinary thermometer scale $(0$ $\left.100^{\circ} \mathrm{C}\right)$. Whereas, the readings of temperature in E.H. during summer (2011), autumn (2011) and winter (2012) were obtained from the calculations of air temperature. The readings of salinity and temperature in E.H. during spring (2012) were obtained by personal communication (Tarek El Gezery). 
Dissolved oxygen was fixed in field and measured according to the modified Winkler's method according to Strickland and Parsons (1972). Nutrient salts (nitrite, nitrate, ammonia, phosphate and silicate) were measured spectrophotometrically in filtered samples, using 6800UV/VIS Spectrophotometer (JENWAY) according to Grasshoff (1976). Oxidizable organic matter was measured according to the method of Carlberg (1972).

\section{Collection of Macroalgae}

Algal samples were collected by hand, washed with seawater at the sampling site to remove the adhered sediments and impurities then separated in polyethylene bags. Samples stored under refrigeration $4^{\circ} \mathrm{C}$. Quick rinsing of the algae with tap water was carried out in the laboratory on the same day to get rid of the remaining impurities and epiphytes. Herbarium sheets with a preliminary identification of separated species were done and/ or preserved in $4 \%$ formalin. Microscopic identification of the investigated algae was carried out according to Aleem (1993).

A total of four algal species were collected from each site at the sub-littoral zone at the depth $(0.5-1 \mathrm{~m})$ representing two algal classes: The Chlorophyceae, which was represented by the order Ulvales comprising one family: Ulvaceae, which was represented by one species; Ulva fasciata Delile, whereas the second class Rhodophyceae was represented by two orders (Corallinales and Gelidiales) comprising one family each (Corallinaceae and Gelidiaceae), respectively. The family Corallinaceae was represented by two species Corallina mediterranea Areschoug and Corallina officinalis Linnaeus. On the other hand, the family Gelidiaceae was represented by the species Pterocladiella capillacea (S.G. Gmelin) Santelices and Hommersand formerly known as Pterocladia capillacea. About 250 gm of each represented species at each study area was air dried to constant weight at room temperature $\left(25^{\circ} \mathrm{C}\right)$. The total average of three replicas and the standard deviation for nutrient contents of each species were calculated.

\section{Extraction and Purification of Total Lipid}

The air dried algal sample was grained manually using mortar and then weighted $(1 \mathrm{~g} \pm 0.001)$ into $100 \mathrm{ml}$ screw top bottles. A total of $50 \mathrm{ml}$ solvent was added in a predetermined sequence, with each sample being sonicated for ease of cell wall rupture and oil extraction by a Lab Sonicator Model Cole-Parmer 8852. The process was accomplished by immersing the bottle containing the algal sample with solvent mixture into the Sonicator at $60^{\circ} \mathrm{C}$ for $15 \mathrm{~min}$.
According to (Folch et al., 1957), lipids were extracted from algae with $30 \mathrm{ml}$ of chloroform/methanol $(2 / 1, \mathrm{v} / \mathrm{v})$ by shaking at $200 \mathrm{rpm}$ and $30^{\circ} \mathrm{C}$. This was followed by the addition of a mixture of methanol/water $(20 \mathrm{ml}, 1: 1, \mathrm{v} / \mathrm{v})$ to achieve a final solvent mixture ratio of $2: 2: 1$ for chloroform: Methanol: Water. The bottles were caped and reshacked for another $30 \mathrm{~min}$. The Supernatants were collected and the residues were re-extracted thrice with $2 \mathrm{ml}$ chloroform/methanol (1/1, v/v) and the combined supernatants were washed with $2 \mathrm{ml}$ of Milli-q water. The lower organic phases were collected after centrifugation; then evaporated till dryness under gentle nitrogen stream.

\section{Determination of Fatty Acids}

The extracted total lipid was transferred to $10 \mathrm{ml}$ clean screw top test tubes, with a fresh prepared solution of methanol: Hydrochloric acid: Chloroform $(10: 1: 1 \mathrm{~V}: \mathrm{V}: \mathrm{V} \mathrm{ml})$ for esterification reaction at $90^{\circ} \mathrm{C}$ for $60 \mathrm{~min}$ (Kumari et al., 2011). The obtained fatty acid methyl esters were subjected to gas liquid chromatographic analysis.

A gas chromatograph Model Hewlett Packard (HP) 6890 GC equipped with a FID (Flame Ionization Detector) was used. The separation column was Fused silica capillary column HP-5 (30 m $\times 0.32 \mathrm{~mm}$ ID $\times 0.25 \mu \mathrm{m}$ film thickness), packed with (5\% diphenyl, $95 \%$ dimethyl polysiloxane) at initial temperature $150^{\circ} \mathrm{C}$ for $2 \mathrm{~min}$. Injector temperature was $220^{\circ} \mathrm{C}$, injection volume was $3 \mu \mathrm{L}$ and detector temperature was $250^{\circ} \mathrm{C}$. Nitrogen was used as the carrier gas and the split ratio was 50:1, while the gas flow was $1 \mathrm{ml}$ $\mathrm{min}^{-1}$. Individual peaks of FAMEs were identified by the comparison of the retention times and equivalent chain length values, using the standard Supelco 37 component FAME Mix, 100mg Neat catalog No. 1819-1AMP with those of authentic standards (Christie, 1988).

\section{Measurements of Nutrient Contents (Phosphorus and Nitrogen)}

For phosphorus analysis, algal sub-samples were dried at $60^{\circ} \mathrm{C}$ to a constant weight, homogenized by crushing each sample in a porcelain pestle and mortar and kept away from metallic materials and dusty conditions to avoid contamination. One gram dry weight of each sample was acid digested in $5 \mathrm{ml}$ concentrated $\mathrm{HNO}_{3}$ in a Teflonlined vessel in a microwave oven in pressure-controlled conditions. Digested samples were filtered through an acidwashed filter (Whatman GF/C) and diluted to $25 \mathrm{ml}$ with double distilled water (Haritonidis and Malea, 
1995; Mohamed and Khaled, 2005). The measurement of phosphorus was based on the reaction of phosphate with molybdate in a strong acidic medium to form a complex (Gamst and Try, 1980). The results of phosphorus were expressed as mg/100 g dry weight.

For measurement of the total organic nitrogen content, the algal samples were dried at $40^{\circ} \mathrm{C}$ to a constant weight. $1.00 \mathrm{~g}$ of the dried sub-sample was completely digested by adding $200 \mathrm{mg}$ of catalyst $\left(\mathrm{K}_{2} \mathrm{SO}_{4}, \mathrm{CuSO}_{4}\right.$ and $\mathrm{SeO}$ in ratio 100:10:1), respectively and $3 \mathrm{ml}$ of concentrated sulfuric acid then diluted to $75 \mathrm{ml}$ with double distilled water (Grasshoff, 1975). The concentration of total nitrogen content in the digested sample was estimated spectrophotometrically at $240 \mathrm{~nm}$ and using ammonium salt as a standard.

\section{Statistical Analysis}

The correlation coefficient between the fatty acids groups with nitrogen and phosphorus content in algal species and with physico-chemical parameters was calculated for each site separately, using the Excel program 13.

\section{Calculation of Biodiesel Physical Properties}

The physical properties of biodiesel products were calculated to investigate the quality of the biodiesel extracted from macroalgal species. The fatty acids methyl ester profiles were used to estimate the Degree of Unsaturation (DU), Long Chain Saturation Factor (LCSF), Cold Filter Plugging Point (CFPP), Iodine Value (IV), Saponification Value (SV), the Cetane Number (CN), kinematic viscosity (v), density $(\rho)$, the Higher Heating Value (HHV), C18:3\% (wt\%) and weight percent of fatty acids with double bond higher than $4 \mathrm{Db} \geq 4$ (wt\%) according to Islam et al. (2013) and Saravanan and Chandrasekar (2013).

\section{Results}

\section{Water Quality}

The water quality of sea water samples collected from Abu Qir Bay (A.Q.) and Eastern Harbor (E.H.) during 2011-2012 is shown in Table 1.

Sea water $\mathrm{pH}$ lied in the alkaline side during the four seasons in the two sites. $\mathrm{pH}$ showed approximately no seasonal variations. The highest $\mathrm{pH}$ in A.Q. and E.H. was recorded during both summer and winter seasons (8.45 and 8.48), respectively. Ismael et al. (2014) recoded the same results; where the $\mathrm{pH}$ values demonstrated negligible variations (8.10-8.30) at both Abu Qir and Eastern Harbor. Water salinity of both sites was lower than that of proper Mediterranean Sea water and ranged between (37.61 to $38.43 \%$ in A.Q.) and (38.11 and $38.35 \%$ in E.H.). The previously recorded salinity by Ismael et al. (2014) ranged between 38.10 and $38.90 \%$ in A.Q. and about $37.4 \%$ at the Eastern Harbor. The recorded salinity reflects the discharged fresh water finger print in both E.H. and A.Q. Bay. The water temperature was minimum during autumn (15.50 and $\left.18.20^{\circ} \mathrm{C}\right)$, while maximum during summer $(26.44$ and $28.90^{\circ} \mathrm{C}$ ) in A.Q. and E.H., respectively. In general, E.H. showed higher temperature compared to A.Q. in all seasons. The same range of temperature was recorded by Ismael et al. (2014) for both sites, since temperature ranged from 18.0 to $29.3^{\circ} \mathrm{C}$.

As indicated from the data presented in the Table 1, dissolved oxygen measurements showed that sea water in A.Q. was well ventilated since the dissolved oxygen content showed relatively higher values (4.07-8.97 $\left.\mathrm{mgO}_{2} / 1\right)$ during all seasons than in E. H., which fluctuated between (3.17-5.23 $\left.\mathrm{mgO}_{2} / 1\right)$.

The oxidizable organic matter ranged between $\left(0.64 \mathrm{mgO}_{2} / 1\right)$ during spring and $\left(1.60 \mathrm{mgO}_{2} / 1\right)$ during winter in A.Q., whereas in Eastern Harbor O.M. ranged between $\left(0.80 \mathrm{mgO}_{2} / 1\right)$ during winter and $(3.90$ $\left.\mathrm{mgO}_{2} / 1\right)$ during summer, with annual average $\left(1.17 \pm 0.40 \mathrm{mgO}_{2} / 1\right)$ for the former and $(2.06 \pm 1.46$ $\left.\mathrm{mgO}_{2} / 1\right)$ for the later.

As far as nutrients are concerned, both nitrite and nitrate showed in A.Q. the maximum concentration during spring and winter (1.03 and $\left.1.63 \mu \mathrm{mol} \mathrm{l}^{-1}\right)$, respectively, whereas, the minimum concentration of the two parameters was recorded during summer $(0.40$ and $0.85 \mu \mathrm{mol} \mathrm{l}^{-1}$ ), respectively. The E. H. showed inverse pattern for nitrite, recording the maximum value during summer $\left(0.95 \mu \mathrm{mol} \mathrm{l}^{-1}\right)$ and the minimum value during spring $\left(0.40 \mu \mathrm{mol} \mathrm{l}^{-1}\right)$, whereas the highest concentration of nitrate was recorded during spring $\left(1.68 \mu \mathrm{mol} \mathrm{l}^{-1}\right)$ and the lowest during winter $\left(0.93 \mu \mathrm{mol}^{-1}\right)$. Inorganic phosphate concentration was relatively low in A.Q. and E.H., except during summer (5.85 and $6.19 \mu \mathrm{mol} \mathrm{l}^{-1}$ ), respectively. Regarding the concentration of inorganic silicate, it was high in A.Q., recording a maximum value of $\left(12.30 \mu \mathrm{mol}^{-1}\right)$ during winter and a low value of $3.29 \mu$ mole $1^{-1}$ during summer. While, the concentration of silicate in E. H. ranged between $\left(3.02 \mu \mathrm{mol} 1^{-1}\right)$ during winter and $\left(9.81 \mu \mathrm{mol} \mathrm{l}^{-1}\right)$ during spring.

\section{The Nutrient Contents (Phosphorus and Nitrogen)}

The contents of the two elements; nitrogen and phosphorus displayed wide spatial and temporal variations in the four algal species (Table 2). The corallinate species, $C$. officinalis recorded the maximum value of nitrogen content in A.Q. during autumn 
$\left(37.67 \pm 5.51 \mathrm{mg} \mathrm{g}^{-1}\right)$ and in E.H. during spring $\left(35.00 \pm 0.00 \mathrm{mg} \mathrm{g}^{-1}\right)$. The lowest value of nitrogen content was recorded in P. capillacea in A.Q. $(6.67 \pm 2.31$ $\left.\mathrm{mg} \mathrm{g}^{-1}\right)$ and in C. officinalis in E.H. $\left(7.07 \pm 0.60 \mathrm{mg} \mathrm{g}^{-1}\right)$ both during winter. The total phosphorus was higher in A.Q. than in Eastern Harbor, with remarkably high concentration in $U$. fasciata in the former site $(1711.00 \pm 21.52 \mathrm{mg} / 100 \mathrm{~g})$, which is approximately two folds of that recorded in $U$. fasciata in E.H. during winter. Whereas, the lowest values were recorded in $C$. mediterranea in A.Q. during spring and C. officinalis in E.H. during autumn and were approximately equal.

Table 1. The physico-chemical parameters in Abu-Qir Bay and Eastern Harbor during the study period (2011-2012) Abu-Qir Bay

\begin{tabular}{|c|c|c|c|c|c|c|c|c|}
\hline $\begin{array}{l}\text { Physico-chemical } \\
\text { parameters }\end{array}$ & $\begin{array}{l}\text { Summer } \\
(2011)\end{array}$ & $\begin{array}{l}\text { Autumn } \\
(2011) \\
\end{array}$ & $\begin{array}{l}\text { Winter } \\
(2012)\end{array}$ & $\begin{array}{l}\text { Spring } \\
(2012) \\
\end{array}$ & $\max$ & $\min$ & average & $\mathrm{SD}$ \\
\hline $\mathrm{pH}$ & 8.45 & 8.38 & 8.45 & 8.22 & 8.45 & 8.22 & & 0.11 \\
\hline Salinity (\%o) & - & - & 37.61 & 38.43 & 38.43 & 37.61 & 38.02 & 0.58 \\
\hline Temperature $\left({ }^{\circ} \mathrm{C}\right)$ & 26.44 & 15.50 & 16.50 & 20.50 & 26.44 & 15.50 & 19.74 & 4.96 \\
\hline D.O. $\left(\mathrm{mgO}_{2} / 1\right)$ & 4.07 & 6.26 & 6.24 & 8.97 & 8.97 & 4.07 & 6.38 & 2.01 \\
\hline $\mathrm{OM}\left(\mathrm{mgO}_{2} / 1\right)$ & 1.30 & 1.12 & 1.60 & 0.64 & 1.60 & 0.64 & 1.17 & 0.40 \\
\hline $\mathrm{NO}_{2}(\mu \mathrm{mol} / 1)$ & 0.40 & 0.75 & 0.55 & 1.03 & 1.03 & 0.40 & 0.68 & 0.27 \\
\hline $\mathrm{NO}_{3}(\mu \mathrm{mol} / 1)$ & 0.85 & 0.98 & 1.63 & 1.03 & 1.63 & 0.85 & 1.12 & 0.35 \\
\hline $\mathrm{PO}_{4}(\mu \mathrm{mol} / \mathrm{l})$ & 5.85 & 0.17 & 0.00 & 0.17 & 5.85 & 0.00 & 1.55 & 2.87 \\
\hline $\mathrm{SiO}_{4}(\mu \mathrm{mol} / \mathrm{l})$ & 3.29 & 4.61 & 12.30 & 9.01 & 12.30 & 3.29 & 7.30 & 4.13 \\
\hline \multicolumn{9}{|c|}{ Eastern Harbor } \\
\hline $\mathrm{pH}$ & 8.47 & 8.19 & 8.48 & 8.12 & 8.48 & 8.12 & & 0.19 \\
\hline Salinity (\%o) & - & - & 38.35 & 38.11 & 38.35 & 38.11 & 38.23 & 0.17 \\
\hline Temperature $\left({ }^{\circ} \mathrm{C}\right)$ & 28.90 & 18.20 & 19.80 & 22.70 & 28.90 & 18.20 & 22.40 & 4.72 \\
\hline D.O. $\left(\mathrm{mgO}_{2} / 1\right)$ & 3.17 & 4.55 & 4.41 & 5.23 & 5.23 & 3.17 & 4.34 & 0.86 \\
\hline $\mathrm{OM}\left(\mathrm{mgO}_{2} / \mathrm{l}\right)$ & 3.90 & 2.56 & 0.80 & 0.96 & 3.90 & 0.80 & 2.06 & 1.46 \\
\hline $\mathrm{NO}_{2}(\mu \mathrm{mol} / 1)$ & 0.95 & 0.93 & 0.45 & 0.40 & 0.95 & 0.40 & 0.68 & 0.30 \\
\hline $\mathrm{NO}_{3}(\mu \mathrm{mol} / \mathrm{l})$ & 1.40 & 1.43 & 0.93 & 1.68 & 1.68 & 0.93 & 1.36 & 0.31 \\
\hline $\mathrm{PO}_{4}(\mu \mathrm{mol} / 1)$ & 6.19 & 0.47 & 0.26 & 0.47 & 6.19 & 0.26 & 1.85 & 2.90 \\
\hline $\mathrm{SiO}_{4}(\mu \mathrm{mol} / \mathrm{l})$ & 5.62 & 4.77 & 3.02 & 9.81 & 9.81 & 3.02 & 5.81 & 2.88 \\
\hline
\end{tabular}

Note: Dissolved Oxygen = D.O.; Oxidizable organic Matter $=\mathrm{OM} ;$ Nitrite $=\mathrm{NO}_{2} ;$ Nitrate $=\mathrm{NO}_{3} ;$ Phosphate $=\mathrm{PO}_{4} ;$ Silicate $=\mathrm{SiO}_{4}$

Table 2. The nutrient content (nitrogen, $\mathrm{mg} / \mathrm{g}$ and phosphorus, $\mathrm{mg} / 100 \mathrm{~g}$ ) and their standard deviation in Abu-Qir Bay and Eastern Harbor during the study period (2011-2012)

\begin{tabular}{|c|c|c|c|c|c|}
\hline \multicolumn{6}{|c|}{ Abu-Qir Bay } \\
\hline Season & Natural content & Summer (2011) & Autumn (2011) & Winter (2012) & Spring (2012) \\
\hline \multirow[t]{2}{*}{ U. fasciata } & Nitrogen & $\mathrm{ND}$ & $*$ & $14.33 \pm 6.51$ & $11.00 \pm 1.00$ \\
\hline & Phosphorus & ND & $*$ & $1711.00 \pm 21.52$ & $337.00 \pm 13.00$ \\
\hline \multirow[t]{2}{*}{ C. mediterranea } & Nitrogen & $*$ & $*$ & $*$ & $32.97 \pm 17.83$ \\
\hline & Phosphorus & $*$ & $*$ & $*$ & $196.33 \pm 4.73$ \\
\hline \multirow[t]{2}{*}{ C. officinalis } & Nitrogen & $*$ & $37.67 \pm 5.51$ & $25.00 \pm 3.46$ & ND \\
\hline & Phosphorus & $*$ & $521.00 \pm 25.51$ & $351.33 \pm 12.34$ & ND \\
\hline \multirow[t]{2}{*}{ P. capillacea } & Nitrogen & $26.33 \pm 5.86$ & ND & $6.67 \pm 2.31$ & $17.67 \pm 4.04$ \\
\hline & Phosphorus & $328.67 \pm 14.22$ & ND & $1363.67 \pm 10.26$ & $324.67 \pm 6.11$ \\
\hline \multicolumn{6}{|c|}{ Eastern Harbor } \\
\hline \multirow[t]{2}{*}{ U. fasciata } & Nitrogen & $12.00 \pm 2.00$ & $8.69 \pm 1.05$ & $8.83 \pm 1.65$ & $10.33 \pm 0.58$ \\
\hline & Phosphorus & $356.33 \pm 23.80$ & $240.33 \pm 19.01$ & $738.33 \pm 3.06$ & $612.00 \pm 21.00$ \\
\hline \multirow[t]{2}{*}{ C. mediterranea } & Nitrogen & ND & $26.93 \pm 2.38$ & $9.9 \pm 2.09$ & $23.50 \pm 1.50$ \\
\hline & Phosphorus & ND & $360.67 \pm 14.01$ & $343.33 \pm 5.86$ & $560.0 \pm 10.82$ \\
\hline \multirow[t]{2}{*}{ C. officinalis } & Nitrogen & ND & $9.11 \pm 1.21$ & $7.07 \pm 0.60$ & $35.00 \pm 0.00$ \\
\hline & Phosphorus & ND & $208.00 \pm 5.29$ & $262.67 \pm 8.62$ & $311.00 \pm 13.89$ \\
\hline \multirow{2}{*}{ P. capillacea } & Nitrogen & $8.90 \pm 1.39$ & $*$ & $*$ & $*$ \\
\hline & phosphorus & $499.33 \pm 15.18$ & $*$ & $*$ & $*$ \\
\hline
\end{tabular}

Note: The * sign means that the species was not found during this season; ND means that the component was not measured. 


\begin{tabular}{|c|c|c|c|c|c|c|c|c|}
\hline \multirow[b]{2}{*}{ Fatty acids } & \multicolumn{2}{|c|}{ Summer (2011) } & \multicolumn{2}{|c|}{ Autumn 2011) } & \multicolumn{2}{|c|}{ Winter (2012) } & \multicolumn{2}{|c|}{ Spring (2012) } \\
\hline & A.Q. & E.H. & A.Q. & E.H. & A.Q. & E.H. & A.Q. & E.H. \\
\hline n-carpoic acid C6:0 & 15.04 & - & $*$ & 1.37 & 27.64 & 5.97 & - & - \\
\hline Caprilic acid C8:0 & 1.47 & - & $*$ & 0.51 & 8.66 & 1.71 & - & 0.09 \\
\hline Capric C10:0 & 4.01 & - & $*$ & 0.55 & 8.38 & 2.44 & - & - \\
\hline Undecanoic acid C11:0 & 1.10 & - & $*$ & 0.35 & 1.97 & 2.41 & - & - \\
\hline Lauric acid C12:0 & 4.81 & - & $*$ & 0.33 & 4.70 & 1.26 & - & 0.41 \\
\hline Tridecanoic acid C13:0 & 1.34 & - & $*$ & 0.28 & 4.10 & 1.46 & - & 0.84 \\
\hline Myrisitic acid C14:0 & 26.32 & 1.62 & $*$ & 5.36 & 90.64 & 20.41 & 0.77 & 16.01 \\
\hline Pentadecanoic acid C15:0 & 16.61 & 1.15 & $*$ & 5.86 & 55.19 & 16.67 & 2.93 & 2.03 \\
\hline Palmatic acid C16:0 & 1222.31 & 145.73 & $*$ & 259.89 & 4025.55 & 1331.97 & 49.41 & 54.32 \\
\hline Margarinic acid C17:0 & 13.75 & - & $*$ & 1.93 & 15.31 & 6.58 & - & 3.81 \\
\hline Stearic acid C18:0 & 20.72 & 2.72 & $*$ & 10.05 & 74.95 & 18.45 & 1.68 & 2.67 \\
\hline Arachidic acid C20:0 & 3.53 & - & $*$ & 0.90 & - & - & - & 1.94 \\
\hline Heneicosanoic acid C21:0 & 20.76 & - & $*$ & 0.92 & 84.12 & 15.25 & - & 2.19 \\
\hline Docosanoic acid C22:0 & 8.54 & - & $*$ & 1.43 & - & - & - & - \\
\hline $\mathrm{C} 23: 0$ & 6.46 & - & $*$ & - & - & - & - & 1.53 \\
\hline $\mathrm{C} 24: 0$ & 0.49 & - & $*$ & 1.61 & - & - & - & - \\
\hline$\Sigma \mathrm{SFA}$ & 1367.26 & 151.22 & $*$ & 291.34 & 4401.22 & 1424.57 & 54.79 & 85.84 \\
\hline Tetradecenoic acid C14:1n-7 & 1.14 & 0.25 & $*$ & 0.54 & 5.87 & 1.98 & - & 0.24 \\
\hline 14, Pentadecenoic acid C15:1 & 2.10 & - & $*$ & 2.21 & 15.78 & 1.32 & 8.29 & 35.38 \\
\hline 9 Hexadecenoic acid C16:1n-7 & 9.80 & 2.29 & $*$ & - & & & - & - \\
\hline Heptadecenoic acid C17:1 & 10.76 & - & $*$ & 15.32 & 208.69 & 90.69 & 446.72 & 93.16 \\
\hline Oleic acid C18:1c n-9 & 64.58 & 14.22 & $*$ & 0.71 & 2.36 & 1.50 & - & 1.12 \\
\hline 5 Ecosenic acid-11Ecosenic acid C20:1n9 & - & - & $*$ & 0.40 & - & - & - & 0.99 \\
\hline 13-Docosenoic acid C22:1n-7 & 4.44 & - & $*$ & 5.12 & 67.39 & 17.90 & - & - \\
\hline$\Sigma$ MUFA & 92.82 & 16.76 & $*$ & 24.30 & 300.09 & 113.39 & 455.01 & 130.89 \\
\hline Linoleic acid C18:2c n-6 & 5.65 & 2.00 & $*$ & - & - & - & - & 0.74 \\
\hline$\gamma$ Linolenic acid C18:3n-6 & 3.05 & - & $*$ & 1.14 & 17.63 & 9.53 & - & 1.07 \\
\hline Ecosadienoic acid C20:2 n-6 & - & - & $*$ & - & - & - & - & - \\
\hline Ecosatrienoic acid C20:3 n-6 & - & - & $*$ & - & - & - & - & 0.46 \\
\hline Arachidonic acid C20:4 n-6 & - & & $*$ & - & - & - & - & - \\
\hline Cis, $5,8,11,14,17$ Ecosapentanoic acid C20:5n-3 & 66.17 & - & $*$ & - & - & - & - & - \\
\hline Docosadienoic acid C22:2 n-6 & - & - & $*$ & - & - & - & - & 2.14 \\
\hline Docosahexanoic acid C22:6n-3 & - & 2.18 & $*$ & - & - & - & - & - \\
\hline$\Sigma$ PUFA & 74.87 & 4.18 & $*$ & 1.14 & 17.63 & 9.53 & - & 4.41 \\
\hline$\Sigma \mathrm{FA}$ & 1534.95 & 172.16 & $*$ & 316.78 & 4718.93 & 1547.50 & 509.80 & 221.14 \\
\hline
\end{tabular}

Note: The * sign means that the species was not found during this season

\section{Fatty Acids Composition}

The four algal species showed completely different pattern of fatty acids composition spatially and temporally. The fatty acids profile in Ulva fasciata revealed higher concentrations in A.Q. bay than that of Eastern Harbor, with a higher value of saturated fatty acid (SFA) than the other two groups during almost all seasons (Table 3). Palmitic acid C16:0 was the dominant fatty acid in the four seasons, recording the highest concentration $\left(4025.55 \mu \mathrm{g} \mathrm{g}^{-1}\right)$ in A.Q. during winter. The second dominant saturated fatty acid was the Myristic acid (C14:0), followed by Stearic acid (C18:0). Considering MUF the Hepatadecenoic acid (C17:1) was dominant, with the highest concentration in A.Q. during spring $\left(446.72 \mu \mathrm{g} \mathrm{g}^{-1}\right)$. The composition of PUFAs group was meager, where many acids were absent. The Cis, 5,8,11,14,17 Ecosapentanoic acid C20:5 was present only in A.Q. during summer (66.17 $\mu \mathrm{g} \mathrm{g}^{-1}$ ), whereas the Docosahexanoic acid C22:6 was present only in E.H. during the same season in very low amount $\left(2.18 \mu \mathrm{g} \mathrm{g}^{-1}\right)$.

The rhodophyte Corallina mediterranea was absent in A.Q. during the three seasons; summer, autumn and winter (Table 4). Whereas, comparing the spring season in the two sites revealed much lower concentrations of fatty acids in A.Q. $\left(25.08 \mu \mathrm{g} \mathrm{g}^{-1}\right)$ than E.H. $\left(360.54 \mu \mathrm{g} \mathrm{g}^{-1}\right)$. Regarding the Eastern Harbor, the two seasons; summer and autumn revealed low concentrations of Fatty acids, with SFA higher 
than MUFAs and PUFAs. In contrast, the two other seasons; winter and spring revealed higher MUFAs than SFA and PUFAs due to the high concentration of Hepatadecenoic acid (C17:1) (801.27 and 281.66 $\mu \mathrm{g}$ $\mathrm{g}^{-1}$ ), respectively. Among the SFA group, palmitic acid was dominant, with highest concentration in Eastern Harbor during winter $\left(203.02 \mu \mathrm{g} \mathrm{g}^{-1}\right)$. Like in $U$. fasciata, the composition of fatty acids of PUFAs group in $C$. mediterranea was meager, where many acids were absent.

The fatty acids profile in Corallina officinalis (Table 5) revealed higher concentrations in A.Q. than that of E.H., except during spring. The Saturated Fatty Acids (SFA) were higher than the other two groups during summer and autumn in both sites. Whereas the opposite pattern was shown during winter and spring in A.Q. and during spring in E.H., where MUFAS were higher than the two other groups; SFA and PUFAs due to the high concentrations of $(\mathrm{C} 17: 1)$ which represent about $78 \%$ of the total fatty acid in A.Q. during winter and $59 \%$ in E. $\mathrm{H}$ during spring. Among the SFA group, palmitic acid was dominant, with highest concentration in Abu-Qir bay during autumn (235.64 $\left.\mathrm{g} \mathrm{g} \mathrm{g}^{-1}\right)$.

The species Pterocladiella capillacea was absent in Eastern harbor during the three seasons; autumn, winter and spring (Table 6). Whereas, comparing the summer season in the two sites revealed much lower concentrations of total fatty acids in E.H. (390.09 $\mu \mathrm{g}$ $\mathrm{g}^{-1}$ ) than in A.Q. (1137.70 $\left.\mu \mathrm{g} \mathrm{g}^{-1}\right)$. Regarding A.Q., the two seasons; summer and autumn revealed high concentrations of Fatty acids, with SFA higher than MUFAs and PUFAs. In contrast, the two other seasons; winter and spring revealed higher MUFAs than SFA and PUFAs due to the high concentration of Heptadecenoic acid (C17:1) (493.87 $\mu \mathrm{g} \mathrm{g}^{-1}$ ) during winter and 9 Hexadecenoic acid C16:1 during spring $\left(458.63 \mu \mathrm{g} \mathrm{g}^{-1}\right)$.

Table 4. The concentration ( $\mu \mathrm{g} / \mathrm{g}$ ) of fatty acids in Corallina mediterranea collected from Abu-Qir Bay (A.Q.) and Eastern Harbor (E.H.) during

\begin{tabular}{|c|c|c|c|c|c|c|c|c|}
\hline \multirow[b]{2}{*}{ Fatty acids } & \multicolumn{2}{|c|}{ Summer (2011) } & \multicolumn{2}{|c|}{ Autumn 2011) } & \multicolumn{2}{|c|}{ Winter (2012) } & \multicolumn{2}{|c|}{ Spring (2012) } \\
\hline & A.Q. & E.H. & A.Q. & E.H. & A.Q. & E.H. & A.Q. & E.H. \\
\hline n-carpoic acid C6:0 & $*$ & - & $*$ & - & $*$ & - & - & 4.70 \\
\hline Caprilic acid C8:0 & * & - & * & 0.46 & * & - & - & 2.07 \\
\hline Lauric acid C12:0 & * & 1.83 & * & - & $*$ & - & - & - \\
\hline Tridecanoic acid C13:0 & * & - & * & - & * & - & - & - \\
\hline Myrisitic acid C14:0 & $*$ & 43.60 & $*$ & 2.83 & $*$ & 11.68 & - & - \\
\hline Pentadecanoic acid C15:0 & $*$ & 4.10 & $*$ & 0.75 & $*$ & - & 0.33 & 9.32 \\
\hline Palmatic acid C16:0 & $*$ & 189.63 & * & 44.71 & $*$ & 203.02 & 0.63 & 4.57 \\
\hline Margarinic acid C17:0 & $*$ & 2.58 & $*$ & - & $*$ & - & - & - \\
\hline Stearic acid $\mathrm{C} 18: 0$ & * & 6.43 & $*$ & 1.98 & $*$ & - & - & - \\
\hline Arachidic acid C20:0 & $*$ & - & $*$ & - & $*$ & - & - & - \\
\hline Heneicosanoic acid C21:0 & $*$ & - & $*$ & - & $*$ & 20.18 & - & 5.09 \\
\hline Docosanoic acid C22:0 & $*$ & - & $*$ & - & $*$ & - & - & - \\
\hline $\mathrm{C} 23: 0$ & $*$ & - & $*$ & & $*$ & 43.81 & - & 7.41 \\
\hline C24:0 & * & - & * & & * & - & - & - \\
\hline$\Sigma$ SFA & * & 248.17 & * & 50.73 & * & 278.69 & 0.96 & 33.16 \\
\hline Tetradecenoic acid C14:1n-7 & * & 10.17 & * & 0.96 & * & 30.07 & 0.42 & 20.01 \\
\hline 14, Pentadecenoic acid C15:1 & * & 4.74 & * & - & * & 70.38 & 1.62 & 22.59 \\
\hline 9 Hexadecenoic acid C16:1n-7 & * & - & * & - & * & - & - & - \\
\hline Heptadecenoic acid C17:1 & * & - & $*$ & 4.42 & $*$ & 801.27 & 21.89 & 281.66 \\
\hline Oleic acid C18:1c n-9 & * & 1.03 & * & - & * & - & - & - \\
\hline 5 Ecosenic acid-11Ecosenic acid C20:1n9 & * & - & * & - & * & - & - & - \\
\hline 13-Docosenoic acid C22:1n-7 & * & - & * & - & * & - & - & - \\
\hline ¿MUFA & $*$ & 15.95 & $*$ & 5.38 & $*$ & 901.72 & 23.93 & 324.26 \\
\hline Linoleic acid C18:2c n-6 & * & 1.39 & * & - & * & - & - & - \\
\hline$\gamma$ Linolenic acid C18:3n-6 & * & - & * & - & * & - & - & - \\
\hline Ecosadienoic acid C20:2 n-6 & * & - & * & - & $*$ & - & - & - \\
\hline Ecosatrienoic acid C20:3n-6 & * & & $*$ & - & $*$ & 5.76 & - & - \\
\hline Arachidonic acid C20:4 n-6 & $*$ & - & * & - & $*$ & - & - & - \\
\hline Cis, $5,8,11,14,17$ Ecosapentanoic acid C20:5n-3 & $*$ & - & * & - & $*$ & - & - & - \\
\hline Docosadienoic acid C22:2 n-6 & $*$ & - & * & - & $*$ & 29.89 & 0.19 & 3.12 \\
\hline Docosahexanoic acid C22:6n-3 & $*$ & 8.48 & * & - & $*$ & - & - & - \\
\hline ¿PUFA & $*$ & 9.87 & $*$ & - & $*$ & 35.65 & 0.19 & 3.12 \\
\hline$\Sigma \mathrm{FA}$ & * & 273.98 & $*$ & 56.11 & $*$ & 1216.06 & 25.08 & 360.54 \\
\hline
\end{tabular}

Note: The $*$ sign means that the species was not found during this season 
Table 5. The concentration $(\mu \mathrm{g} / \mathrm{g}$ ) of fatty acids in Corallina officinalis collected from Abu-Qir Bay (A.Q.) and Eastern Harbor (E.H.) during (2011-2012)

\begin{tabular}{|c|c|c|c|c|c|c|c|c|}
\hline \multirow[b]{2}{*}{ Fatty acids } & \multicolumn{2}{|c|}{ Summer (2011) } & \multicolumn{2}{|c|}{ Autumn (2011) } & \multicolumn{2}{|c|}{ Winter (2012) } & \multicolumn{2}{|c|}{ Spring (2012) } \\
\hline & A.Q. & E.H. & A.Q. & E.H. & A.Q. & E.H. & A.Q. & E.H. \\
\hline n-carpoic acid C6:0 & $*$ & - & 2.08 & 0.72 & - & - & - & 3.50 \\
\hline Caprilic acid C8:0 & $*$ & - & 0.79 & 0.20 & - & - & - & 1.24 \\
\hline Capric C10:0 & $*$ & - & - & 0.55 & - & - & - & - \\
\hline Undecanoic acid C11:0 & $*$ & - & - & - & - & - & - & - \\
\hline Lauric acid C12:0 & $*$ & - & - & 0.76 & - & - & - & - \\
\hline Tridecanoic acid C13:0 & $*$ & 1.32 & - & 0.21 & - & - & - & - \\
\hline Myrisitic acid C14:0 & $*$ & 25.68 & 16.84 & 5.43 & 1.08 & 2.16 & - & 3.17 \\
\hline Pentadecanoic acid C15:0 & $*$ & 9.13 & 11.15 & 2.54 & - & 2.57 & - & 1.69 \\
\hline Palmatic acid C16:0 & $*$ & 82.62 & 235.64 & 134.85 & 38.96 & 71.27 & 0.82 & 88.17 \\
\hline Margarinic acid C17:0 & $*$ & - & - & 3.56 & 0.89 & - & - & - \\
\hline Stearic acid C18:0 & $*$ & - & 21.60 & - & 1.68 & - & - & 10.26 \\
\hline Arachidic acid C20:0 & $*$ & - & - & - & - & - & - & 7.08 \\
\hline Heneicosanoic acid C21:0 & $*$ & - & - & - & - & - & - & 6.06 \\
\hline Docosanoic acid C22:0 & $*$ & - & 9.75 & 15.39 & - & 15.28 & - & - \\
\hline $\mathrm{C} 23: 0$ & $*$ & - & - & - & 6.87 & - & 0.76 & 23.98 \\
\hline $\mathrm{C} 24: 0$ & $*$ & - & - & 23.40 & - & 21.78 & - & - \\
\hline$\Sigma \mathrm{SFA}$ & $*$ & 118.75 & 297.85 & 187.61 & 49.48 & 113.06 & 1.58 & 145.15 \\
\hline Tetradecenoic acid C14:1n-7 & $*$ & 20.14 & 9.25 & 5.56 & 4.02 & 3.70 & 0.50 & 26.28 \\
\hline 14, Pentadecenoic acid C15:1 & $*$ & - & - & 5.05 & - & - & 2.17 & 24.07 \\
\hline 9 Hexadecenoic acid C16:1n-7 & $*$ & 4.80 & - & 3.84 & - & - & - & - \\
\hline Heptadecenoic acid C17:1 & $*$ & - & 48.16 & 5.28 & 200.33 & 11.44 & 21.26 & 316.60 \\
\hline Oleic acid C18:1c n-9 & $*$ & 2.27 & 0.40 & - & - & - & 0.17 & - \\
\hline 5 Ecosenic acid-11Ecosenic acid C20:1n9 & $*$ & - & - & - & - & - & - & - \\
\hline 13-Docosenoic acid C22:1n-7 & $*$ & - & 6.33 & - & - & - & - & - \\
\hline$\Sigma$ MUFA & $*$ & 27.21 & 64.14 & 19.73 & 204.35 & 15.14 & 24.10 & 366.95 \\
\hline Linoleic acid C18:2c n-6 & $*$ & 2.26 & - & - & - & - & - & 2.15 \\
\hline$\gamma$ Linolenic acid C18:3n-6 & $*$ & - & - & - & 0.47 & - & - & - \\
\hline Ecosadienoic acid C20:2 n-6 & $*$ & - & - & - & - & - & - & 5.93 \\
\hline Ecosatrienoic acid C20:3 n-6 & $*$ & - & - & - & - & & - & 4.88 \\
\hline Arachidonic acid C20:4 n-6 & $*$ & - & - & - & - & - & - & - \\
\hline Cis, $5,8,11,14,17$ Ecosapentanoic acid C20:5n-3 & $*$ & - & - & - & - & - & - & - \\
\hline Docosadienoic acid C22:2 n-6 & $*$ & - & - & - & 3.08 & - & 0.34 & 12.02 \\
\hline Docosahexanoic acid C22:6n-3 & $*$ & 18.18 & - & - & - & - & - & - \\
\hline$\Sigma$ PUFA & $*$ & 20.44 & - & - & 3.55 & - & - & 24.98 \\
\hline$\Sigma$ FA & $*$ & 166.40 & 361.99 & 207.34 & 257.38 & 128.20 & 26.02 & 537.08 \\
\hline
\end{tabular}

Note: The * sign means that the species was not found during this season

Among the SFA group, palmitic acid was dominant, with highest concentration in Abu- Qir bay during autumn (1525.72 $\left.\mu \mathrm{g} \mathrm{g}^{-1}\right)$, followed by the Myristic acid (C14:0) and Stearic acid (C18:0). The same pattern of the other algae was followed by $P$. capillacea, where PUFAs group contributed by very low percentage to the total fatty acids.

\section{Statistical Analysis}

The correlation coefficient between the fatty acids groups in the species (U. fasciata, P. capillacea and C. officinalis) in A.Q. and the species (U. fasciata, C.mediterranea and C. officinalis) in E.H. with nutrient content in seaweeds and with physicochemical parameters revealed different effect of these parameters on fatty acids groups (Table 7).

\section{Calculation of Biodiesel Physical Properties}

The results of the physico-chemical properties of produced biodiesel are shown in Table 8, including the degree of saturation of fatty acids methyl ester (DU), Long Chain Saturation Factor (LCSF), Cold Filter Plugging Point (CFPP), Iodine Value (IV), Saponification Value (SV), the Cetane Number (CN), Kinematic viscosity (v), density $(\rho)$, the Higher Heating Value (HHV), C18:3 (wt $\%$ ) and double bond $\mathrm{Db} \geq 4(\mathrm{wt} \%$ ). 
Nayrah Abdel Naby Shaltout and Nihal Galal Shams El-Din / American Journal of Environmental Sciences 2015, 11 (6): 402.419 DOI: 10.3844/ajessp.2015.402.419

\begin{tabular}{|c|c|c|c|c|c|c|c|c|}
\hline \multirow[b]{2}{*}{ Fatty acids } & \multicolumn{2}{|c|}{ Summer (2011) } & \multicolumn{2}{|c|}{ Autumn (2011) } & \multicolumn{2}{|c|}{ Winter (2012) } & \multicolumn{2}{|c|}{ Spring (2012) } \\
\hline & A.Q. & E.H. & A.Q. & E.H. & A.Q. & E.H. & A.Q. & E.H. \\
\hline n-carpoic acid C6:0 & 1.39 & - & 3.95 & $*$ & - & * & - & $*$ \\
\hline Caprilic acid C8:0 & 0.48 & - & 1.55 & $*$ & - & * & 0.61 & $*$ \\
\hline Capric C10:0 & 0.23 & - & - & $*$ & - & $*$ & 0.20 & $*$ \\
\hline Undecanoic acid C11:0 & - & - & - & $*$ & - & $*$ & 1.16 & $*$ \\
\hline Lauric acid C12:0 & 2.09 & 1.62 & 1.86 & $*$ & - & $*$ & - & $*$ \\
\hline Tridecanoic acid C13:0 & 0.93 & 1.05 & 1.33 & * & - & $*$ & 2.29 & $*$ \\
\hline Myrisitic acid C14:0 & 97.45 & 52.21 & 179.37 & $*$ & 1.02 & $*$ & 6.05 & $*$ \\
\hline Pentadecanoic acid C15:0 & 5.65 & 6.79 & 9.14 & $*$ & 10.78 & $*$ & 0.12 & $*$ \\
\hline Palmatic acid C16:0 & 833.39 & 251.84 & 1525.72 & $*$ & 14.78 & * & 63.29 & $*$ \\
\hline Margarinic acid C17:0 & 4.90 & 6.31 & 16.15 & $*$ & - & * & - & $*$ \\
\hline Stearic acid C18:0 & 28.27 & 4.97 & 46.76 & $*$ & 1.26 & * & 3.65 & $*$ \\
\hline Arachidic acid C20:0 & 1.60 & - & - & $*$ & - & * & 0.93 & $*$ \\
\hline Heneicosanoic acid C21:0 & 34.48 & - & - & $*$ & 1.98 & * & - & * \\
\hline Docosanoic acid C22:0 & 2.37 & - & - & * & - & * & - & * \\
\hline $\mathrm{C} 23: 0$ & 2.20 & - & - & * & - & * & 5.14 & $*$ \\
\hline $\mathrm{C} 24: 0$ & - & - & - & $*$ & - & * & - & $*$ \\
\hline$\Sigma$ SFA & 1015.43 & 324.79 & 1785.83 & $*$ & 29.82 & $*$ & 83.44 & $*$ \\
\hline Tetradecenoic acid C14:1n-7 & 44.50 & 21.32 & 38.87 & $*$ & 13.24 & * & 18.06 & $*$ \\
\hline 14, Pentadecenoic acid C15:1 & 1.70 & 2.07 & 32.56 & $*$ & 59.48 & * & 44.74 & $*$ \\
\hline 9 Hexadecenoic acid C16:1n-7 & 8.41 & 5.89 & - & $*$ & - & * & 458.63 & $*$ \\
\hline Heptadecenoic acid C17:1 & 3.52 & 10.66 & 252.59 & $*$ & 493.87 & * & - & $*$ \\
\hline Oleic acid C18:1c n-9 & 12.70 & 4.14 & - & * & - & * & - & $*$ \\
\hline 5 Ecosenic acid-11Ecosenic acid C20:1n9 & 1.30 & - & - & * & - & * & 1.72 & * \\
\hline 13-Docosenoic acid C22:1n-7 & 6.42 & - & - & * & - & * & 1.24 & $*$ \\
\hline ¿MUFA & 78.55 & 44.08 & 324.02 & $*$ & 566.59 & * & 524.39 & $*$ \\
\hline Linoleic acid C18:2c n-6 & 27.01 & 10.53 & - & * & - & * & - & * \\
\hline$\gamma$ Linolenic acid C18:3n-6 & 1.43 & 1.56 & - & * & - & * & - & $*$ \\
\hline Ecosadienoic acid C20:2 n-6 & - & - & - & $*$ & - & * & - & $*$ \\
\hline Ecosatrienoic acid C20:3 n-6 & - & - & - & $*$ & - & * & 1.38 & $*$ \\
\hline Arachidonic acid C20:4 n-6 & 5.16 & - & - & $*$ & - & * & - & * \\
\hline Cis, 5,8,11,14,17Ecosapentanoic acid C20:5n-3 & 4.94 & 1.51 & - & $*$ & - & $*$ & - & * \\
\hline Docosadienoic acid C22:2 n-6 & 2.53 & - & - & $*$ & 0.92 & $*$ & 3.52 & $*$ \\
\hline Docosahexanoic acid C22:6n-3 & 2.65 & 7.62 & - & $*$ & - & * & - & $*$ \\
\hline ¿PUFA & 43.72 & 21.22 & - & $*$ & 0.92 & * & 4.90 & * \\
\hline$\Sigma F A$ & 1137.70 & 390.09 & 2109.85 & * & 597.33 & * & 612.73 & $*$ \\
\hline
\end{tabular}

Note: The * sign means that the species was not found during this season

Table 7. Correlation coefficient between the fatty acids groups with nutrient content in algae (nitrogen and phosphorus) and physico-chemical parameters of the surrounding seawater in the two studied sites

\begin{tabular}{|c|c|c|c|c|c|c|c|c|c|c|c|c|}
\hline \multirow[b]{2}{*}{ Parameters } & \multicolumn{4}{|c|}{ U. fasciata A.Q. } & \multicolumn{4}{|c|}{ P. Capillacea A.Q. } & \multicolumn{4}{|c|}{ C. officinalis A.Q. } \\
\hline & $\Sigma$ SFA & $\Sigma$ MUFA & ¿PUFA & $\Sigma$ FA & $\Sigma$ SFA & $\Sigma$ MUFA & $\Sigma$ PUFA & $\Sigma$ FA & $\Sigma$ SFA & $\Sigma$ MUFA & ¿PUFA & $\Sigma \mathrm{FA}$ \\
\hline Nitrogen & 0.58 & 0.86 & -0.42 & 0.64 & -0.29 & -0.49 & 0.84 & -0.47 & 0.87 & 0.57 & 0.29 & 1.00 \\
\hline Phosphorus & 0.91 & 0.46 & -0.20 & 0.92 & -0.72 & 0.54 & -0.21 & -0.68 & 0.87 & 0.58 & 0.29 & 1.00 \\
\hline $\mathrm{NO}_{2}$ & -0.56 & 0.55 & -0.81 & -0.51 & -0.21 & 0.59 & -0.63 & -0.08 & 0.12 & -0.19 & -0.25 & 0.00 \\
\hline $\mathrm{NO}_{3}$ & 0.87 & 0.39 & -0.31 & 0.88 & -0.60 & 0.73 & -0.54 & -0.50 & -0.12 & 0.97 & 0.99 & 0.41 \\
\hline $\mathrm{PO}_{4}$ & -0.06 & -0.39 & 0.97 & -0.08 & 0.24 & -0.89 & 1.00 & 0.04 & -0.40 & -0.56 & -0.40 & -0.61 \\
\hline $\mathrm{SiO}_{4}$ & 0.65 & 0.75 & -0.48 & 0.70 & -0.83 & 0.92 & -0.62 & -0.71 & -0.31 & 0.79 & 0.86 & 0.16 \\
\hline $\mathrm{OM}$ & 0.83 & -0.39 & 0.42 & 0.78 & 0.03 & -0.12 & 0.15 & 0.00 & 0.04 & 0.67 & 0.66 & 0.38 \\
\hline Chlorophyll & -0.06 & -0.38 & 0.96 & -0.08 & 0.23 & -0.88 & 1.00 & 0.03 & -0.41 & -0.56 & -0.40 & -0.62 \\
\hline $\mathrm{pH}$ & 0.63 & -0.62 & 0.60 & 0.57 & 0.27 & -0.43 & 0.38 & 0.20 & 0.11 & 0.40 & 0.38 & 0.30 \\
\hline $\mathrm{DO}_{2}$ & -0.30 & 0.74 & -0.82 & -0.23 & -0.47 & 0.78 & -0.70 & -0.33 & -0.05 & 0.04 & 0.03 & -0.01 \\
\hline Salinity & 0.42 & 0.94 & -0.47 & 0.49 & -0.93 & 0.89 & -0.52 & -0.83 & -0.50 & 0.51 & 0.64 & -0.14 \\
\hline \multirow[t]{2}{*}{ Temperature } & -0.39 & 0.47 & 0.37 & -0.33 & -0.35 & -0.24 & 0.56 & -0.48 & -0.74 & -0.71 & -0.47 & -0.97 \\
\hline & \multicolumn{4}{|c|}{ U. fasciata E.H. } & \multicolumn{4}{|c|}{ C. mediterranea E.H. } & \multicolumn{4}{|c|}{ C. officinalis E.H. } \\
\hline Parameters & $\Sigma$ SFA & $\Sigma$ MUFA & $\Sigma$ PUFA & $\Sigma F A$ & $\Sigma$ SFA & $\Sigma$ MUFA & $\Sigma$ PUFA & $\Sigma F A$ & $\Sigma$ SFA & $\Sigma$ MUFA & $\Sigma$ PUFA & $\Sigma \mathrm{FA}$ \\
\hline Nitrogen & -0.56 & -0.28 & -0.13 & -0.56 & -0.90 & -0.14 & -0.51 & -0.35 & 0.23 & 0.96 & 0.48 & 0.96 \\
\hline Phosphorus & 0.64 & 0.91 & 0.89 & 0.70 & -0.67 & 0.34 & -0.12 & 0.10 & 0.25 & 0.54 & -0.20 & 0.53 \\
\hline $\mathrm{NO}_{2}$ & -0.43 & -0.99 & -0.66 & -0.51 & 0.04 & -0.79 & -0.45 & -0.65 & 0.36 & -0.61 & -0.13 & -0.51 \\
\hline $\mathrm{NO}_{3}$ & -0.95 & -0.09 & -0.75 & -0.92 & -0.79 & -0.72 & -0.92 & -0.82 & 0.49 & 0.69 & 0.71 & 0.78 \\
\hline $\mathrm{PO}_{4}$ & -0.39 & -0.62 & -0.15 & -0.43 & 0.46 & -0.50 & -0.13 & -0.30 & -0.41 & -0.29 & 0.47 & -0.31 \\
\hline $\mathrm{SiO}_{4}$ & -0.73 & 0.35 & -0.36 & -0.67 & -0.67 & -0.34 & -0.63 & -0.47 & 0.17 & 0.94 & 0.84 & 0.95 \\
\hline $\mathrm{OM}$ & -0.53 & -0.93 & -0.56 & -0.59 & 0.17 & -0.79 & -0.42 & -0.62 & 0.09 & -0.47 & 0.18 & -0.41 \\
\hline Chlorophyll & -0.41 & -0.61 & -0.16 & -0.45 & 0.44 & -0.51 & -0.15 & -0.31 & -0.41 & -0.26 & 0.50 & -0.28 \\
\hline $\mathrm{pH}$ & 0.58 & -0.22 & 0.62 & 0.54 & 0.99 & 0.37 & 0.75 & 0.58 & -0.77 & -0.69 & -0.23 & -0.79 \\
\hline $\mathrm{DO}_{2}$ & 0.04 & 0.72 & -0.01 & 0.10 & -0.70 & 0.31 & -0.15 & 0.07 & 0.43 & 0.67 & -0.05 & 0.69 \\
\hline Salinity & 0.58 & 0.97 & 0.77 & 0.64 & 0.11 & 0.88 & 0.60 & 0.77 & -0.45 & 0.47 & 0.02 & 0.36 \\
\hline Temperature & -0.44 & -0.30 & -0.02 & -0.45 & 0.38 & -0.36 & -0.12 & -0.21 & -0.55 & 0.07 & 0.75 & 0.01 \\
\hline
\end{tabular}

Note: The bold values mean significant correlation 


\begin{tabular}{|c|c|c|c|c|c|c|c|c|c|c|c|c|c|c|c|c|}
\hline \multicolumn{2}{|l|}{ Algae } & DU & LCSF & CFPP & $\begin{array}{l}\text { IV } \\
\left(\mathrm{gI}_{2} 100 \mathrm{~g}^{-1} \text { fat }\right)\end{array}$ & $\begin{array}{l}\mathrm{SV} \\
\left(\mathrm{mgKOHg}^{-1}\right)\end{array}$ & $\mathrm{CN}$ & $\begin{array}{l}\text { TFA wt } \\
(\mu \mathrm{g} / \mathrm{g})\end{array}$ & $\begin{array}{l}\text { SFAs } \\
(\%)\end{array}$ & $\begin{array}{l}\text { MUFA } \\
(\%)\end{array}$ & $\begin{array}{l}\text { PUFA } \\
(\%)\end{array}$ & $\begin{array}{l}\text { (v) } \\
\left(\mathrm{mm}^{2} \mathrm{~s}^{-1}\right)\end{array}$ & $\begin{array}{l}(\rho) \\
\left(\mathrm{g} \mathrm{m}^{-3}\right)\end{array}$ & $\begin{array}{l}\mathrm{HHV} \\
\left(\mathrm{MJ} \mathrm{g}^{-1}\right)\end{array}$ & $\begin{array}{l}\mathrm{C} 18: 3 \\
(\mathrm{wt} \%) \\
\end{array}$ & $\begin{array}{l}\mathrm{Db} \geq \\
4(\mathrm{wt} \%)\end{array}$ \\
\hline \multicolumn{2}{|c|}{ Biodiesel Standard } & - & - & $\leq 5 / \leq-20$ & $\leq 120$ & - & $\geq 51$ & - & - & - & - & $3.5-5.0$ & $0.86-0.9$ & NA & $\leq 12$ & $\leq 1$ \\
\hline \multicolumn{2}{|c|}{$\begin{array}{l}\text { Biodiesel Standard } \\
\text { ASTM D6751-02 }\end{array}$} & - & - & NA & NA & - & $\geq 47$ & - & - & - & - & $1.9-6.0$ & NA & NA & - & - \\
\hline \multicolumn{2}{|c|}{$\min / \max$} & $\max$ & $\max$ & $\max$ & $\max$ & $\max$ & $\min$ & $\min$ & $\min$ & $\max$ & $\max$ & $\max$ & $\max$ & $\min$ & $\max$ & $\max$ \\
\hline \multicolumn{2}{|c|}{ Threshold value } & - & - & 5.00 & 120.00 & - & 47.0 & - & - & - & - & - & 0.90 & - & 12.00 & 1.00 \\
\hline \multirow[t]{2}{*}{ Summer } & $\mathrm{AQ}$ & 15.80 & 9.77 & 14.21 & 23.71 & 206.71 & 68.96 & 1534.95 & 89.08 & 6.05 & 4.88 & 4.27 & 0.86 & 39.41 & 0.20 & 4.31 \\
\hline & $\mathrm{EH}$ & 14.59 & 9.25 & 12.60 & 13.31 & 204.86 & 72.62 & 172.16 & 87.84 & 9.74 & 2.43 & 4.42 & 0.87 & 39.99 & 0.00 & 0.00 \\
\hline \multirow[t]{2}{*}{ Autumn } & AQ & & & & & & & & & & & & & & & \\
\hline & EH & 8.39 & 11.77 & 20.49 & 7.62 & 206.65 & 72.41 & 316.78 & 91.97 & 7.67 & 0.36 & 4.38 & 0.86 & 39.49 & 0.36 & 0.00 \\
\hline \multirow[t]{2}{*}{ Winter } & $\mathrm{AQ}$ & 7.11 & 9.32 & 12.82 & 6.49 & 207.41 & 72.36 & 4718.93 & 93.27 & 6.36 & 0.37 & 4.35 & 0.86 & 39.46 & 0.37 & 0.00 \\
\hline & EH & 8.56 & 9.20 & 12.44 & 8.01 & 207.11 & 72.15 & 1547.50 & 92.06 & 7.33 & 0.62 & 4.34 & 0.86 & 39.49 & 0.62 & 0.00 \\
\hline \multirow[t]{2}{*}{ Spring } & $\mathrm{AQ}$ & 89.25 & 1.13 & -12.91 & 80.42 & 199.59 & 59.17 & 509.80 & 10.75 & 89.25 & 0.00 & 4.09 & 0.86 & 39.61 & 0.00 & 0.00 \\
\hline & EH & 63.18 & 3.94 & -4.11 & 58.49 & 204.16 & 62.52 & 221.14 & 38.82 & 59.19 & 1.99 & 4.07 & 0.87 & 39.75 & 0.48 & 0.00 \\
\hline \multicolumn{17}{|c|}{ Corallina mediterranea } \\
\hline \multirow[t]{2}{*}{ Summer } & $\mathrm{AQ}$ & & & & & & & & & & & & & & & \\
\hline & $\mathrm{EH}$ & 13.03 & 8.09 & 8.95 & 13.73 & 210.66 & 71.32 & 273.98 & 90.58 & 5.82 & 3.60 & 4.21 & 0.89 & 40.35 & 0.00 & 0.00 \\
\hline \multirow[t]{2}{*}{ Autumn } & $\mathrm{AQ}$ & & & & & & & & & & & & & & & \\
\hline & EH & 9.59 & 9.73 & 14.10 & 8.89 & 208.69 & 71.36 & 56.11 & 90.41 & 9.59 & 0.00 & 4.24 & 0.86 & 39.45 & 0.00 & 0.00 \\
\hline \multirow[t]{2}{*}{ Winter } & $\mathrm{AQ}$ & & & & & & & & & & & & & & & \\
\hline & $\mathrm{EH}$ & 80.01 & 1.67 & -11.23 & 72.33 & 198.87 & 62.44 & 1216.06 & 22.92 & 74.15 & 2.93 & 4.34 & 0.88 & 40.41 & 0.00 & 0.00 \\
\hline \multirow[t]{2}{*}{ Spring } & AQ & 96.93 & 0.25 & -15.69 & 86.70 & 199.23 & 57.61 & 25.08 & 3.83 & 95.41 & 0.76 & 4.02 & 0.87 & 39.80 & 0.00 & 0.00 \\
\hline & $\mathrm{EH}$ & 91.67 & 0.13 & -16.08 & 82.37 & 199.83 & 57.31 & 360.54 & 9.20 & 89.94 & 0.87 & 3.97 & 0.87 & 39.57 & 0.00 & 0.00 \\
\hline \multicolumn{17}{|c|}{ Corallina officinalis } \\
\hline \multirow[t]{2}{*}{ Summer } & AQ & & & & & & & & & & & & & & & \\
\hline & $\mathrm{EH}$ & 40.92 & 4.97 & -0.88 & 43.33 & 209.63 & 69.70 & 166.40 & 71.36 & 16.35 & 12.28 & 4.29 & 0.95 & 42.98 & 0.00 & 0.00 \\
\hline \multirow[t]{2}{*}{ Autumn } & AQ & 17.72 & 13.53 & 26.04 & 16.02 & 206.27 & 70.77 & 361.99 & 82.28 & 17.72 & 0.00 & 4.35 & 0.86 & 39.47 & 0.00 & 0.00 \\
\hline & EH & 9.52 & 40.21 & 109.85 & 9.31 & 199.51 & 74.91 & 207.34 & 90.48 & 9.52 & 0.00 & 4.92 & 0.86 & 39.64 & 0.00 & 0.00 \\
\hline \multirow[t]{2}{*}{ Winter } & AQ & 82.15 & 1.84 & -10.70 & 73.85 & 194.42 & 61.60 & 257.38 & 19.22 & 79.40 & 1.38 & 4.27 & 0.87 & 40.03 & 0.18 & 0.00 \\
\hline & EH & 11.81 & 57.42 & 163.91 & 11.07 & 191.50 & 77.96 & 128.20 & 88.19 & 11.81 & 0.00 & 5.45 & 0.86 & 39.91 & 0.00 & 0.00 \\
\hline \multirow[t]{2}{*}{ Spring } & AQ & 92.62 & 0.32 & -15.49 & 86.28 & 194.66 & 58.65 & 26.02 & 6.07 & 92.62 & 0.00 & 4.16 & 0.87 & 40.01 & 0.00 & 0.00 \\
\hline & EH & 77.63 & 3.92 & -4.18 & 70.48 & 193.33 & 62.29 & 537.08 & 27.03 & 68.32 & 4.65 & 4.33 & 0.88 & 40.23 & 0.00 & 0.00 \\
\hline \multicolumn{17}{|c|}{ Pterocladiella capillacea } \\
\hline Summer & $\mathrm{AQ}$ & 14.59 & 9.02 & 11.86 & 15.16 & 206.92 & 70.84 & 1137.70 & 89.25 & 6.90 & 3.84 & 4.30 & 0.87 & 39.62 & 0.13 & 0.89 \\
\hline & EH & 22.18 & 7.09 & 5.81 & 22.70 & 210.05 & 69.00 & 390.09 & 83.26 & 11.30 & 5.44 & 4.13 & 0.88 & 39.99 & 0.40 & 0.39 \\
\hline Autumn & $\begin{array}{l}\mathrm{AQ} \\
\mathrm{EH}\end{array}$ & 15.36 & 8.34 & 9.72 & 14.25 & 208.88 & 70.16 & 2109.85 & 84.64 & 15.36 & 0.00 & 4.19 & 0.86 & 39.44 & 0.00 & 0.00 \\
\hline Winter & $\begin{array}{l}\mathrm{AQ} \\
\mathrm{EH}\end{array}$ & 95.16 & 0.35 & -15.37 & 86.85 & 201.64 & 57.15 & 597.33 & 4.99 & 94.85 & 0.15 & 3.96 & 0.87 & 39.57 & 0.00 & 0.00 \\
\hline Spring & $\begin{array}{l}\mathrm{AQ} \\
\mathrm{EH}\end{array}$ & 87.18 & 1.48 & -11.82 & 82.96 & 208.24 & 55.70 & 612.73 & 13.62 & 85.58 & 0.80 & 3.68 & 0.87 & 39.45 & 0.00 & 0.00 \\
\hline
\end{tabular}

\section{Discussion}

Fatty acids are influenced by environmental factors such as temperature, light, $\mathrm{pH}$, salinity and nutrients (Aknin et al., 1990; Dembitsky et al., 1991). The temperature is one of the most important environmental factors that influence algal growth rate, cell size, biochemical composition and nutrient requirements (Juneja et al., 2013). Kalacheva et al. (2002) stated that, lipid composition is one of the most commonly observed changes with temperature shift. Moreover, Thompson et al. (1992) reported that Myristic acids (14:0) increased with higher temperature while PUFA were consistently higher at lower temperature. The reported data for the present study in E.H. followed exactly Thompson et al. (1992), where all algae showed the highest PUFA content during winter. In addition, Myristic acid in $C$. mediterranea and C. officinalis in E.H. showed the highest concentration during summer except $U$. fasciata which showed inverse pattern. This could be interpreted based on the findings of Converti et al. (2009) who found that the effect of temperature on lipid composition varied specifically. Concerning the fatty acid content in algal species in A.Q., it showed different patterns in contrast to Thompson et al. (1992). Statistically, fatty acids groups were insignificantly correlated with temperature except the total fatty acids $\Sigma$ FA in C. officinalis in A.Q. Bay $(\mathrm{r}=-0.97)$.

Another important factor is $\mathrm{pH}$, since it determines the solubility and availability of $\mathrm{CO}_{2}$ and essential nutrients and because it can have a significant impact on algal metabolism (Chen and Durbin, 1994). Alkaline $\mathrm{pH}$ increases the flexibility of the cell wall, resulting in an increase in triglyceride accumulation but a decrease in membrane-associated polar lipids (Guckert and Cooksey, 1990). On the other hand, acidic conditions can alter nutrient uptake (Gensemer et al., 1993). Tatsuzawa et al. (1996) observed that acidic conditions cause an increase in 
saturated fatty acid content, which reduces membrane fluidity and inhibits high proton concentrations. However, in the present study, $\mathrm{pH}$ lied in the alkaline side at both sites and it was found that $\mathrm{pH}$ was only positively correlated with SFA in $C$. mediterranea in E.H., which antagonize with Tatsuzawa et al. (1996).

Salinity is another factor that alters the biochemical composition of algal cells. Exposing algae to lower or higher salinity levels than their natural levels can change growth rate and alter composition such as lipid content. $\mathrm{Xu}$ and Beardall (1997) found that a species of Dunaliella exhibited an increase in SFA and MUFA fatty acids with an increase in $\mathrm{NaCl}$ concentration. In the present study, salinity influenced positively the MUFA in $U$. fasciata in both sites A.Q. and E.H. ( $\mathrm{r}=0.97$ and $0.94, \mathrm{p} \leq 0.05)$, respectively, which agreed with the results of $\mathrm{Xu}$ and Beardall (1997), whereas it influenced negatively the SFA in P. capillacea $(\mathrm{r}=-0.93, \mathrm{p} \leq 0.05)$.

Nitrogen is an important macronutrient for growth and metabolism of algal cells. It is a fundamental element for the formation of proteins and nucleic acids (Harris, 1986) and is available in seawater in the form inorganic nitrogenous compounds (nitrite, nitrate and ammonia). However, $\mathrm{Hu}$ (2004) reported that nitrogen accounts for $7-20 \%$ of cell dry weight. Inorganic nitrogen taken up by algae is rapidly assimilated into biochemically active compounds and recycled within cells to meet changing physiological needs (Vergara et al., 1995). Major effects of nitrogen deficiency include the enhanced biosynthesis and accumulation of lipids (Demirbas, 2010) and triglycerides (Stephenson et al., 2010) with a concomitant reduction in protein content (Heraud et al., 2005). This, in turn, results in a higher lipid/protein ratio at the expense of growth rate (Converti et al., 2009). In the present study, the nitrogen content in seaweeds was lower in the two sites than the range of $\mathrm{Hu}$ (2004), ranging from 0.67 to $3.77 \%$ in A.Q. and from 0.71 to $3.50 \%$ in E.H. However, nitrogen in tissue was positively correlated with $\Sigma \mathrm{FA}$ in $C$. officinalis in A.Q. and E.H. and with $\Sigma$ MUFA in the same species in E.H. Concerning the inorganic nitrogen; nitrate was positively correlated with

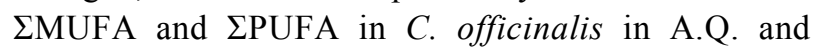
negatively correlated with $\Sigma$ SFA and $\Sigma$ FA in $U$. fasciata and with $\Sigma$ PUFA in C. mediterranea, whereas nitrite influenced only negatively $\Sigma$ MUFA in $U$. fasciata in E.H.

Phosphorus is another important macronutrient, which is available as reactive phosphate in sea water.
It is required for normal growth and development of algal cells (Hu, 2004). Also, it is a key component of phospholipids and an integral part of essential molecules such as ATP and a part of the backbone of DNA and RNA (Harris, 1986). It has been shown that phosphorus, rather than nitrogen, is the primary limiting nutrient for algae in many natural environments (Larned, 1998). Reitan et al. (1994) reported that phosphorus limitation leads to accumulation of lipids and reduces the synthesis of n3 PUFA. According to the Björnsäter and Wheeler's classification (1990) of macroalgal nutrient status based on $\mathrm{N}: \mathrm{P}$ ratio of tissues, a $\mathrm{N}: \mathrm{P}$ ratio $<16$ indicates N-limitation; a N:P ratio $16-24$ indicates $\mathrm{N}$ and $\mathrm{P}$ sufficiency (no limitation) and $\mathrm{N}: \mathrm{P}>24$ indicates P-limitation. Applying this classification to our data, we could conclude that they are N-limited in both sites, since in general the ratios in A.Q. did not exceed 8:1, except in $C$. mediterranea during spring (16.79). On the other hand, this ratio in E.H. did not exceed 7.5, except in C. officinalis (11.2) during spring. Nascimento et al. (2014) recorded a higher range of $\mathrm{N}: \mathrm{P}$ ratio in tissue from 10:1 to $34: 1$ with absence of seasonal variations of the two nutrients. The seasonal and spatial variations in tissue nutrients were observed in the present study, with the maximum value of $\mathrm{N}$ and $\mathrm{P}$ recorded in A.Q. during autumn and winter and in E.H. during winter and spring, respectively. Phosphorus was positively correlated with $\Sigma$ SFA and $\Sigma$ FA of $U$. fasciata and with $\Sigma$ FA in C. officinalis in A.Q., indicating the role of phosphorus in the synthesis of fatty acids. On the other hand, the inorganic phosphorus was correlated positively with SPUFA in $U$. fasciata and $P$. capillacea in A.Q., whereas, inorganic phosphorus had no any influence on the fatty acids groups in the algal species of E.H.

Noticeably, the present study showed great seasonal, specifical and spatial variations for lipid profile. Thus, screening for the optimum value for biodiesel properties before its processing is very important to define the suitable site, season and species to obtain the optimum biodiesel.

It was observed that the fuel properties of biodiesel play a significant role in the combustion process and delay period, which in turn affect the engine performance (Sivaramakrishnan and Ravikumar, 2012). The Cetane Number (CN) of the fuel is such an important parameter which is responsible for the delay period. A fuel of higher cetane number gives lower delay period and provides smoother engine operation. Both the British biodiesel standard EN (14214) and 
the American biodiesel standard ASTM D6751-02 stated that a cetane number $\mathrm{CN}$ should be $(\geq 51$ and $\geq 47$ ), respectively. In fact, biodiesel has a higher $\mathrm{CN}$ than petrodiesel because of its higher oxygen content (Sivaramakrishnan and Ravikumar, 2012). In the present study, the biodiesel has a $\mathrm{CN}$ (based on molecular weight and degree of unsaturation) that exceeds 52 and its value varied within species and seasons. The highest $\mathrm{CN}$ was recorded in C. officinalis in E.H. during winter (77.96), whereas the lowest $\mathrm{CN}$ (55.70) was recorded in P. capillacea in A.Q. during spring. Factors that affect the $\mathrm{CN}$ in the biodiesel are the number of carbon atoms of the original fatty acids, the number of double bonds, the high amounts of SFA in addition to the type of feed stock (Gopinath et al., 2009; Tesfa et al., 2010).

The degree of saturation plays a significant role in fuel properties. It has been suggested that, the higher the degree of unsaturation of the FAMEs of a biodiesel, the higher the tendency of the biodiesel to oxidize (Islam et al., 2013). It also influences largely the kinematic viscosity (v), Cold Filter Plugging Point (CFPP) and density ( $\rho)$ (Moser, 2014). The shorter and more unsaturated chains increase the viscosity and flow characteristics at low temperatures, which are undesirable characteristics of fuels. To obtain a biodiesel with appropriate quality characteristics, an appropriate ratio between saturated and unsaturated fatty acids should be maintained (Knothe, 2005). In the present study, the percentage of saturated FAs was almost high, against MUFA\% and PUFA\%. The maximum percentage was recorded in $U$. fasciata (93.27\%) in A.Q. during winter, whereas, the lowest percentage was recorded in C. mediterranea $(3.83 \%)$ in A.Q. during spring.

Kinematic viscosity is a measure of the internal friction or resistance of oil to flow and is the most important physical property of biodiesel since it affects the operation of fuel injection equipment, particularly at low temperatures when the increase in viscosity affects the fluidity of the fuel. High viscosity leads to poorer atomization of the fuel spray and less accurate operation of the fuel injectors (Sivaramakrishnan and Ravikumar, 2012). Kinematic viscosity limits are set by international biodiesel standards to be in the ranges of 1.9-6.0 mm $\mathrm{m}^{-1}$ and $3.5-5.0 \mathrm{~mm}^{2} \mathrm{~s}^{-1}$ as per ASTM6751-02 and EN 14214, respectively (Islam et al., 2013). In the present study, the produced biodiesel from all macroalgal species were in the prescribed viscosity range with 3.68-5.54 $\mathrm{mm}^{2} \mathrm{~s}^{-1}$, therefore meeting both standards. Sivaramakrishnan and Ravikumar (2012) recorded viscosity values of biodiesel between 3.6 and $5.7 \mathrm{~mm}^{2}$ $\mathrm{s}^{-1}$, whereas, Islam et al. (2013) recorded in some microalgal species viscosity range between 3.44 and $4.20 \mathrm{~mm}^{2} \mathrm{~s}^{-1}$.

It is noteworthy to mention that, the fuel injection system supplies fuel by volume not by mass which means denser biodiesel will be injected with greater mass into the combustion chamber, consequently affecting the stoicheometric ratio of air and fuel (Ng et al., 2012). Therefore, density ( $\rho)$ is another important parameter for biodiesel quality, for which a standard value has been set at $0.86-0.90 \mathrm{~g} \mathrm{~cm}^{-3}$ according to EN 14214. Our results are consistent with the standard value, where the density of produced biodiesel in the collected algae ranged between 0.86 and $0.89 \mathrm{~g} \mathrm{~cm}^{-3}$ except for $C$. officinalis during summer at A.Q. where the extracted biodiesel recorded a density of $0.95 \mathrm{~g} \mathrm{~cm}^{-3}$.

The Heat of combustion refers to the measure of energy content in the fuel (Sivaramakrishnan and Ravikumar, 2012). Heating value of fuels is an important measure of its releasing energy for producing work. According to Sivaramakrishnan and Ravikumar (2012), the lower heating value of biodiesel is attributed to the decrease in engine power and the energy content of oils which depends on the place where they are grown, the season, the composition and other factors. In fact, the $\mathrm{HHV}$ values in the present study (39.41-42.98 $\mathrm{MJ} \mathrm{kg}^{-1}$ ) were closed to that of biodiesel blend Jatropha (39.1741.52 $\mathrm{MJ} \mathrm{kg}^{-1}$ ) and blend Karanja (35.9-41.5 $\mathrm{MJ} \mathrm{kg}^{-1}$ ) fuel, where they exceeded the values of oils obtained from some crops (Sivaramakrishnan and Ravikumar, 2012) and the values of some microalgae (36.6-40.4 $\mathrm{MJ} \mathrm{kg}^{-1}$ ) (Islam et al., 2013). Ramirez-Verduzco et al., (2012) found that the range of regular biodiesel was between 39.8 and $40.4 \mathrm{MJ} / \mathrm{kg}$, which is normally 10 to $12 \%$ less than obtained for petroleum-derived diesel (46 $\mathrm{MJ} \mathrm{kg}^{-1}$ ).

The Iodine Index (IV) refers to the tendency of biodiesel to react with oxygen at room temperature. The low iodine index indicates less susceptibility to oxidation by oxygen (Duarte and Maugeri, 2014), which is desirable for biodiesel. According to the biodiesel standard EN (14214), iodine value is $\leq 120$ $\mathrm{gI}_{2} 100 \mathrm{~g}^{-1}$ fat. In the present study, all IV values are within the limits of the standards. It ranged between minimum $6.49 \mathrm{gI}_{2} 100 \mathrm{~g}^{-1}$ fat for $U$. faciata in A.Q. during autumn to maximum $86.85 \mathrm{gI}_{2} 100 \mathrm{~g}^{-1}$ fat for $P$. capillacea in A.Q. during winter.

Regarding $\mathrm{C} 18: 3 \mathrm{wt} \%$ and the weight percentage of fatty acid esters containing number of double bonds 
higher than 4, they are of the most important property that govern the biodiesel stability. A limit of $12 \mathrm{wt} \%$ for this FAME has been set in the European B100 biodiesel standard (EN 14214), which also limits the amount of FAMEs with four or more double bonds to 1 wt\% (Barabás and Todoruţ, 2011). Therefore, polyunsaturated fatty acid content of the biomass, as well as the weighted degree of unsaturation developed by Ramos et al. (2009) and the predictive fuel stability calculated from only two FAME contents, linoleate (C18:2) and linolenate (C18:3) can serve as indirect estimates of biodiesel oxidative stability. All algae from this study were much lower than the EN14214 threshold of $12 \mathrm{wt} \% \mathrm{C} 18: 3$. Whereas, the values of $w t \%$ of $\geq$ four double bonds complied with that of biodiesel standard EN (14214) $(\leq 1)$, except the values of $U$. fasciata in E.H. during summer was (4.31). The results recorded revealed that the produced biodiesel of all algal species during all seasons at both sites is of highest oxidative stability.

On the other hand, the values of Cold Filter Plugging Point (CFPP) in the present study complied almost with the value set by biodiesel standard EN (14214), ranging from $\leq 5 / \leq-20$, with some exceptions, especially the highly exclusive values in C. officinalis in the E.H. during autumn (109.85) and winter (163.91). In fact, Islam et al. (2013) mentioned that CFPP is of low importance in subtropical and tropical climates. It is noteworthy to mention, that our study region is considered as subtropical, therefore, the weighting of the CFPP parameter was of less importance than other criteria. Based on all these biofuel criteria, the green alga $U$. fascaita collected from A.Q. during winter was the most suitable choice for biodiesel production.

\section{Conclusion and Recommendations}

From the present study it is concluded that, the green alga Ulva fasciata collected from Abu Qir Bay during winter can be considered as the most suitable and promising source for biodiesel production. Regarding the biodiesel produced from Corallina officinalis, it showed the highest biodiesel quality but its quantity is very low. So it can be used as a blending to the petroleum diesel to increase its quality, oxidative stability and octane number.

Based on all the obtained results we can recommend the following:

- In order to produce a high quality and a large quantity of biodiesel it is recommended to cultivate Ulva fasciata during winter
- In order to produce biodiesel of very high quality to be used as an additive to improve petroleum diesel quality, it is better to cultivate Corallina officinalis during winter.

\section{Acknowledgment}

We are greatly indebted to Dr. Tarek El Gezery, Lecturer in Marine physics Laboratory, Marine Environment Department, National Institute of Oceanography and Fisheries, Alexandria, Egypt, who helped us and supplied us with the data of salinity and temperature in this work.

\section{Author's Contributions}

All authors equally contributed in this work.

\section{Ethics}

This article is original and contains unpublished material. The corresponding author confirms that all of the other authors have read and approved the manuscript and no ethical issues involved.

\section{References}

Abdallah, M.A.M., 2013. The potential of different bio adsorbents for removing phenol from its aqueous solution. Environ. Monitor. Assessment, 185: 6495-6503.

DOI: $10.1007 / \mathrm{s} 10661-012-3041-y$

Abishek, M.P., J. Patel and A.P. Rajan, 2014. Algae oil: A sustainable renewable fuel of future. Biotechnol. Res. Int., 2014: 272814-272821. DOI: $10.1155 / 2014 / 272814$

Afify, A.M.R., E.A. Shalaby and S.M.M. Shanab, 2010. Enhancement of biodiesel production from different species of algae. Grasas Y Aceites, 61: 416-422. DOI: 10.3989/gya.021610

Aknin, M., J. Mirailles and J.M. Kornprobst, 1990. Sterol and fatty acid distribution in red algae from the Senegalese coast. Comparative Biochem. Physiol., 96B: 559-563. DOI: 10.1016/0305-0491(90)90056-Y

Aleem, A.A., 1993. The Marine Algae of Alexandria, Egypt. 1st Edn., Alexandria, ISBN-10: 9770052817 , pp: 138 .

Barabás, I. and I.A. Todoruţ, 2011. Biodiesel Quality, Standards and Properties. In: Biodiesel-Quality, Emissions and By-Products, Montero, G. and M. Stoytcheva (Eds.), InTech., Rijeka, Croacia, pp: 3-28. 
Björnsäter, B.R. and P.A. Wheeler, 1990. Effect of nitrogen and phosphorus supply on growth and tissue composition of Ulva fenestrata and enteromorpha intestinalis (Ulvales, Chlorophyta). J. Phycol., 26: 603-611. DOI: $10.1111 / \mathrm{j} .0022-3646.1990 .00603 . \mathrm{x}$

Carlberg, S.R., 1972. New Baltic manual, intern. Coun. For the Explo. Of the Sea. Cooperative Res. Rep. Series A N. 29, Copenhagen.

Chen, C.Y. and E.G. Durbin, 1994. Effects of pH on the growth and carbon uptake of marine phytoplankton. Mar. Ecol.-Prog. Ser., 109: 83-94. DOI: $10.3354 /$ meps 109083

Christie, W.W., 1988. Equivalent chain-lengths of methyl ester derivatives of fatty acids on gas chromatography a reappraisal. J. Chromatogr. A, 447: 305-314.

DOI: 10.1016/0021-9673(88)90040-4

Converti, A., A.A. Casazza, E.Y. Ortiz, P. Perego and M. del Borghi, 2009. Effect of temperature and nitrogen concentration on the growth and lipid content of Nannochloropsis oculata and Chlorella vulgaris for biodiesel production. Chem. Eng. Process, 48: 1146-1151.

DOI: 10.1016/J.CEP.2009.03.006

Dembitsky, V.M., E.E. Pechenkina-Shubina and O.A. Rosentsvet, 1991. Glycolipids and fatty acids of some seaweeds and marine grasses from the Black Sea. Phytochemistry, 31: 2279-2283.

Demirbas, A., 2009. Production of biodiesel from algae oils. Energy Sources Part A: Recovery Utiliz. Environ. Effects, 31: 163-168. DOI: $10.1080 / 15567030701521775$

Demirbas, A., 2010. Use of algae as biofuel sources. Energy Convers. Manag., 51: 2738-2749. DOI: 10.1016/j.enconman.2010.06.010

Dickson, A.G., C.L. Sabine and J.R. Christian, 2007. Guide to Best Practices for Ocean $\mathrm{CO}_{2}$ Measurements. 1st Edn., PICES Special Publication, pp: 191.

Duarte, S.H. and F. Maugeri, 2014. Prediction of quality properties for biodiesel production by oleaginous yeast cultivated in pure and raw glycerol. Chemical Engineering Transactions, 37: 463-468. DOI: 10.3303/CET1437078

El-Kassas, H.Y. and A.A. Attia, 2014. Bactericidal application and cytotoxic activity of biosynthesized silver nanoparticles with an extract of the red seaweed Pterocladiella capillacea on the $\mathrm{HepG}_{2}$ cell line. Asian Pacific J. Cancer Prevent., 15: 1229-1306.

DOI: 10.7314/APJCP.2014.15.3.1299
El-Sarraf, W.M., 1995. Heavy metal content in some marine algae from Alexandria, Egypt. Bull. Fac. Sci., Alexandria Univ., 35: 475-484.

El-Sayed, M.K. and A. Khadr, 1999. Technical Report on Geological Setting of the Eastern Harbour. 1st Edn., Alexandria, Egypt, pp: 60.

El-Tawil, B.A.H. and A.N. Khalil, 1983. Chemical constituents of some algal species from Abu-Qir Bay. Egypt. J. Fac. Mar. Sci., 3: 85-94.

Folch, J., M. Lees and G.H. Sloane-Stanley, 1957. A simple method for the isolation and purification of total lipides from animal tissues, J. Biol. Chem., 226: 497-509. PMID: 13428781

Gamst, O. and K. Try, 1980. Determination of serumphosphate without deproteinization by ultraviolet spectrophotometry of the phosphomolybdic acid complex. Scandinavian J. Clin. Laboratory Investigat., 40: 483-486.

DOI: $10.3109 / 00365518009101872$

Gensemer, R.W., R.E.H. Smith and H.C. Duthie, 1993. Comparative effects of $\mathrm{pH}$ and aluminum on silica-limited growth and nutrient uptake in Asterionella ralfsii var. Americana (Bacillariophyceae). J. Phycol., 29: 36-44. DOI: 10.1111/j.1529-8817.1993.tb00277.x

Goh, C.S. and K.T. Lee, 2010. A visionary and conceptual macroalgae-based Third-Generation Bioethanol (TGB) biorefinery in Sabah, Malaysia as an underlay for renewable and sustainable development. Renewable Sustainable Energy Rev., 14: 842-848. DOI: 10.1016/j.rser.2009.10.001

Gopinath, A., S. Puhan and G. Nagarajan, 2009. Relating the cetane number of biodiesel fuels to their fatty acid composition: A critical study. Proc. Inst. Mech. Eng. Part D J. Automob. Eng., 223: 565-583. DOI: 10.1243/09544070JAUTO950

Grasshoff, K., 1975. The Hydrochemistry of Landlocked Basins and Fjords. In: Chemical Oceanography, Riley, J.P. and G. Skirrow (Eds.), Academic Press, London, pp: 568-574.

Grasshoff, K., 1976. Methods of Seawater Analysis. 1st Edn., Verlage Chemie, New York, pp: 317.

Guckert, J.B. and K.E. Cooksey, 1990. Triglyceride accumulation and fatty acid profile changes in Chlorella (chlorophyta) during high $\mathrm{pH}$-induced cell cycle inhibition. J. Phycol., 26: 72-79. DOI: $10.1111 /$ j.0022-3646.1990.00072.x

Haritonidis, S. and P. Malea, 1995. Seasonal and local variation of $\mathrm{Cr}, \mathrm{Ni}$ and $\mathrm{Co}$ concentrations in Ulva rigida C. Agardh and Enteromorpha linza (Linnaeus) from Thermaikos Gulf, Greece. J. Environ. Poll., 89: 319-327. DOI: 10.1016/0269-7491(94)00070-T 
Harris, G.P., 1986. Phytoplankton Ecology: Structure, Function and Fluctuation. 1st Edn., Chapman and Hall, New York, ISBN-10: 0412306905, pp: 394.

Heraud, P., B.R. Wood, M.J. Tobin, J. Beardall and D. McNaughton, 2005. Mapping of nutrient-induced biochemical changes in living algal cells using synchrotron infrared microspectroscopy. FEMS Microbiol. Lett., 249: 219-225.

DOI: 10.1016/j.femsle.2005.06.021

$\mathrm{Hu}$, Q., 2004. Environmental Effects on Cell Composition. In: Handbook of Microalgal Culture: Biotechnology and Applied Phycology, Richmond, A. (Ed.), Blackwell Publishing Ltd, Oxford, pp: 83-93.

Hughes, D.A., M.S. Kelly, K.D. Black and M.S. Stanley, 2012. Biogas from Macroalgae: Is it time to revisit the idea? Biotechnol. Biofuels, 5: 86-86. DOI: 10.1186/1754-6834-5-86

Islam, M.A., M. Magnusson, R.J. Brown, G.A. Ayoko and M.N. Nabi et al., 2013. Microalgal species selection for biodiesel production based on fuel properties derived from fatty acid profiles. Energies, 6: 5676-5702.

DOI: $10.3390 /$ en6115676

Ismael, A.A., 2012. Benthic bloom of cyanobacteria associated with fish mortality in Alexandria waters. Egypt. J. Aquatic Res., 38: 241-247.

DOI: $10.1016 /$ j.ejar.2013.01.001

Ismael, A.A.H., E.A. Mohamed, M.M. El-Sheikh and W.H. Hegazy, 2014. Ecological distribution of harmful epiphytic Oscillatoriales in Alexandria coast, Egypt, with special reference to DNA identification. J. Coastal Life Med., 2: 274-280. DOI: 10.12980/JCLM.2.2014J51

John, R. and G. Anisha, 2011. Macroalgae and their potential for biofuel. Nut. Nat. Res., 6: 1-15.

Juneja, A., R.M. Ceballos and G.S. Murthy, 2013. Effects of environmental factors and nutrient availability on the biochemical composition of algae for biofuels production: A review. Energies, 6: 4607-4638. DOI: 10.3390/en6094607

Kalacheva, G., N. Zhila, T. Volova and M. Gladyshev, 2002. The effect of temperature on the lipid composition of the green alga Botryococcus. Microbiology, 71: 286-293.

DOI: $10.1023 / \mathrm{A}: 1015898426573$

Knothe, G., 2005. Dependence of biodiesel fuel properties on the structure of fatty acid alkyl esters. Fuel Process. Technol., 86: 1059-1070. DOI: 10.1016/j.fuproc.2004.11.002
Kumari, P., C. Reddy and B. Jha, 2011. Comparative evaluation and selection of a method for lipid and fatty acid extraction from macroalgae. Anal. Biochem., 415: 134-144.

DOI: $10.1016 /$ J.AB.2011.04.010

Larned, S.T., 1998. Nitrogen-versus phosphorus-limited growth and sources of nutrients for coral reef macroalgae. Marine Biol., 132: 409-421.

DOI: $10.1007 / \mathrm{s} 002270050407$

Le Goff, C., J.F. Kaux, L. Leroy, J. Pincemail and J.P. Chapelle et al., 2013. Fatty acids and associated cardiovascular risk. Rapport d'activités - CHU Université de Liège, pp: 14.

Maceiras, R., M. Rodriguez, A. Cancela, S. Urrejola and A. Sanchez, 2011. Macroalgae: Raw material for biodiesel production. Applied Energy, 88: 3318-3323. DOI: 10.1016/j.apenergy.2010.11.027

Marchetti, J.M., V.U. Miguel and A.F. Errazu, 2007. Possible methods for biodiesel production. Renew. Sust. Energy Rev., 11: 1300-1311. DOI: 10.1016/j.rser.2005.08.006

Matloub, A.A., M. El-Sherbini, I.H. Borai, M.K. Ezz and M.Z. Rizk et al., 2013. Assessment of antihyperlipidemic effect and physco-chemical characterization of water soluble polysaccharides from Ulva Fasciata delile. J. Applied Sci. Res., 9: 2983-2993.

Mohamed, L.A. and A. Khaled, 2005. Comparative study of heavy metal distribution in some coastal seaweeds of Alexandria, Egypt. Chem. Ecol., 21: 181-189. DOI: 10.1080/02757540500151614

Moser, B.R., 2014. Impact of fatty ester composition on low temperature properties of biodiesel-petroleum diesel blends. Fuel, 115: 500-506.

DOI: 10.1016/J.FUEL.2013.07.075

Muralidhar, A.P., K. Sayamala, C. Prakash, C. Kalidas and R.P. Naik, 2010. Comparative studies on fatty acid composition of three marine macroalgae collected from Mandapam Region: South East Coast of India. J. World Applied Sci., 11: 958-965.

Nascimento, A., C. Coelho-Gomes, E. Barbarino and S.O. Lourenço, 2014. Temporal variations of the chemical composition of three seaweeds in two tropical coastal environments. Open J. Marine Sci., 4: 118-139. DOI: 10.4236/ojms.2014.42013

$\mathrm{Ng}$, J.H., H.K. Ng and S. Gan, 2012. Characterisation of engine-out responses from a light-duty diesel engine fuelled with Palm Methyl Ester (PME). Applied Energy, 90: 58-67.

DOI: 10.1016/J.APENERGY.2011.01.028 
Pienkos, P.T. and A. Darzins, 2009. The promise and challenges of microalgal-derived biofuels. Biofuels Bioprod. Biorefin., 3: 431-440.

DOI: $10.1002 / \mathrm{bbb} .159$

Polat, S. and Y. Ozogul, 2013. Seasonal proximate and fatty acid variations of some seaweeds from the northeastern Mediterranean coast. Oceanologia, 55: 375-391.

DOI: $10.5697 /$ oc. $55-2.375$

Pulz, O. and W. Gross, 2004. Valuable products from biotechnology of microalgae. Applied Microbiol. Biotechnol., 65: 635-648. DOI: $10.1007 / \mathrm{s} 00253-004-1647-\mathrm{x}$

Ramirez-Verduzco, L.F., J.E. Rodríguez-Rodríguez and A.R. Jaramillo-Jacob, 2012. Predicting cetane number, kinematic viscosity, density and higher heating value of biodiesel from its fatty acid methyl ester composition. Fuel, 91: 102-111. DOI: 10.1016/j.fuel.2011.06.070

Ramos, M.J., C.M. Fernandez, A. Casas, L. Rodriguez and A. Perez, 2009. Influence of fatty acid composition of raw materials on biodiesel properties. J. Bioresour. Technol., 100: 261-268. DOI: $10.1016 /$ j.biortech.2008.06.039

Reitan, K.I., J.R. Rainuzzo and Y. Olsen, 1994. Effect of nutrient limitation on fatty acid and lipid content of marine microalgae. J. Phycol., 30: 972-979. DOI: $10.1111 / \mathrm{j} .0022-3646.1994 .00972 . x$

Renaud, S.M. and J.T. Luong-Van, 2006. Seasonal variation in the chemical composition of tropical Australian marine macroalgae. J. Applied Phys., 18: 381-387. DOI: 10.1007/s10811-006-9034-x

Saravanan, S. and S. Chandrasekar, 2013. Predictive equations for viscosity and cetane number of biodiesel. German J. Renewable Sustainable Energy Res., 1: 23-28.

Shams El-Din, N.G., A.M. Amer and M.A. Abdallah, 2007. Study of natural components in some marine macroalgae in relation to nutrients along Alexandrial Coast, Egypt. Egypt. J. Aquat. Res., 33: 87-112.

Shams El-Din, N.G. and Z.M. El-Sherif, 2012. Nutritional value of some algae from the northwestern Mediterranean coast of Egypt. J. Applied Phycol., 24: 613-626.

DOI: $10.1007 / \mathrm{s} 10811-012-9831-3$

Shams El-Din, N.G., L.I. Mohamedein and K.M. ElMoselhy, 2014. Seaweeds as bioindicators of heavy metals off a hot spot area on the Egyptian Mediterranean coast during 2008-2010. J. Environ. Monitor. Assessment, 186: 5865-5881.

DOI: $10.1007 / \mathrm{s} 10661-014-3825-3$
Singh, J. and S. Gu, 2010. Commercialization potential of microalgae for biofuels production. Renew. Sustain. Energy Rev., 14: 2596-2610. DOI: 10.1016/J.RSER.2010.06.014

Sivaramakrishnan, K. and P. Ravikumar, 2012. Determination of cetane number of biodiesel and it's influence on physical properties. ARPN J. Eng. Applied Sci., 7: 205-211.

Stephenson, A.L., J.S. Dennis, C.J. Howe, S.A. Scott and A.G., Smith, 2010. Influence of nitrogen-limitation regime on the production by Chlorella vulgaris of lipids for biodiesel feedstocks. Biofuels, 1: 47-58. DOI: $10.4155 / \mathrm{bfs} .09 .1$

Strickland, J.D.M. and T.R. Parsons, 1972. A practical Handbook of Seawater Analysis. 2nd Edn., Fisheries Research Board of Canada, Ottawa, pp: 310.

Surendhiran, D. and M. Vijay, 2014. Effect of various pretreatment for extracting intracellular lipid from Nannochloropsis oculata under nitrogen replete and depleted conditions. ISRN Chem. Eng., 2014: 536310-536318. DOI: 10.1155/2014/536310

Tadros, A.B., F.A. Zaghloul, H.R. Zaki, A.E. Abaas and M.M. Kandeel, 2006. Impact of coatings containing algae on some seawater parameters and epiphytes plankton. Egypt. J. Aquatic Res., 32: $24-37$.

Tatsuzawa, H., E. Takizawa, M. Wada and Y. Yamamoto, 1996. Fatty acid and lipid composition of the acidophilic green alga Chlamydomonas sp. 1. J. Phycol., 32: 598-601. DOI: $10.1111 /$ j.0022-3646.1996.00598.x

Tesfa, B., R. Mishra, F. Gu and N. Powles, 2010. Prediction models for density and viscosity of biodiesel and their effects on fuel supply system in CI engines. Renewable Energy, 35: 2752-2760. DOI: 10.1016/J.RENENE.2010.04.026

Thompson, P.A., M. Guo and P.J. Harrison, 1992. Effects of variation in temperature. I. On the biochemical composition of eight species of marine phytoplankton. J. Phycol., 28: 481-488. DOI: $10.1111 / \mathrm{j} .0022-3646.1992 .00481 . \mathrm{x}$

Trivedi, N., V. Gupta, C.R.K. Reddy and J. Bhavanath, 2013. Enzymatic hydrolysis and production of bioethanol from common macrophytic green alga Ulva fasciata Delile. Bioresource Technol., 150: 106-112. DOI: 10.1016/j.biortech.2013.09.103

Vergara, J., K. Bird and F. Niell, 1995. Nitrogen assimilation following $\mathrm{NH}_{4}^{+}$pulses in the red alga Gracilariopsis lemaneiformis: Effect on C metabolism. Mar. Ecol. Prog. Ser., 122: 253-263. DOI: $10.3354 /$ meps 122253 
Wassef, E.A., A.M. El-Sayed and E.M., Sakr, 2013. Pterocladia (Rhodophyta) and Ulva (Chlorophyta) as feed supplements for European seabass, Dicentrarchus labrax L., Fry. J. Applied Phycol., 25: 1369-1376. DOI: 10.1007/s10811-013-9995-5

$\mathrm{Xu}$, X.Q. and J. Beardall, 1997. Effect of salinity on fatty acid composition of a green microalga from an antarctic hypersaline lake. Phytochemistry, 45: 655-658. DOI: 10.1016/s0031-9422(96)00868-0
Yang, B., K.O. Kalimo, R.L. Tahvonen, L.M. Mattila and J.K. Katajisto et al., 2000. Effect of dietary supplementation with sea buckthorn (Hippophaë rhamnoides) seed and pulp oils on the fatty acid composition of skin glycerophospholipids of patients with atopic dermatitis. J. Nutr. Biochem., 11: 338-340.

DOI: $10.1016 / \mathrm{S} 0955-2863(00) 00088-7$ 